\title{
AN APPLIED MATHEMATICAL EXCURSION THROUGH LYAPUNOV INEQUALITIES, CLASSICAL ANALYSIS AND DIFFERENTIAL EQUATIONS
}

\author{
ANTONIO CAÑADA AND SALVADOR VILLEGAS
}

\begin{abstract}
Several different problems make the study of the so called Lyapunov type inequalities of great interest, both in pure and applied mathematics. Although the original historical motivation was the study of the stability properties of the Hill equation (which applies to many problems in physics and engineering), other questions that arise in systems at resonance, crystallography, isoperimetric problems, Rayleigh type quotients, etc. lead to the study of $L_{p}$ Lyapunov inequalities $(1 \leq p \leq \infty)$ for differential equations. In this work we review some recent results on these kinds of questions which can be formulated as optimal control problems. In the case of Ordinary Differential Equations, we consider periodic and antiperiodic boundary conditions at higher eigenvalues and by using a more accurate version of the Sturm separation theory, an explicit optimal result is obtained. Then, we establish Lyapunov inequalities for systems of equations. To this respect, a key point is the characterization of the best $L^{p}$ Lyapunov constant for the scalar given problem, as a minimum of some especial (constrained or unconstrained) variational problems defined in appropriate subsets of the usual Sobolev spaces. For Partial Differential Equations on a domain $\Omega \subset \mathbb{R}^{N}$, it is proved that the relation between the quantities $p$ and $N / 2$ plays a crucial role in order to obtain nontrivial $L_{p}$ Lyapunov type inequalities (which are called Sobolev inequalities by many authors). This fact shows a deep difference with respect to the ordinary case. Combining the linear results with Schauder fixed point theorem, we can obtain some new results about the existence and uniqueness of solutions for resonant nonlinear problems for ODE or PDE, both in the scalar case and in the case of systems of equations.
\end{abstract}

\section{INTRODUCTION}

The Hill equation

$$
u^{\prime \prime}(t)+a(t) u(t)=0, t \in \mathbb{R}
$$

2000 Mathematics Subject Classification. 34B05, 34B15, 34C10, 35J25, 35J20, 49J15, 49J20.

Key words and phrases. Lyapunov inequalities, boundary value problems, resonance, stability, ordinary differential equations, partial differential equations.

The authors have been supported by the Ministry of Education and Science of Spain (MTM2008.00988) and by Junta de Andalucia (FQM116). 
where the function $a$ satisfies

$$
a: \mathbb{R} \rightarrow \mathbb{R} \text { is } T \text { - periodic and } a \in L^{1}(0, T),
$$

models many phenomena in applied sciences ([17, [21, [31]). In a broader sense, Hill's equation connotes the class of homogeneous, linear, second order differential equations with real and periodic coefficients. In the preface to the book on Hill's equation, by Magnus and Winkler [31], we can read:

"There exist hundreds of applications of Hill's equation to problems in engineering and physics, including problems in mechanics, astronomy, the theory of electric circuits, of the electric conductivity of metals, of the cyclotron and new applications are continually discovered".

In what follows, we denote by $L_{T}(\mathbb{R}, \mathbb{R})$ the set of functions $a(\cdot)$ satisfying (1.2).

The study of stability properties of (1.1) is of special interest. Whenever all solutions of (1.1) are bounded, we say that (1.1) is stable; otherwise we say that it is unstable. Floquet theory assures that such stability properties depend on characteristic multipliers, which are defined using any fundamental matrix of the given equation. This important theoretical result requires the knowledge of all solutions of (1.1).

In early twentieth century Lyapunov proved ([30, [31]) that if

$$
0 \prec a, \int_{0}^{T} a(t) d t \leq \frac{4}{T},
$$

then (1.1) is stable. Here, for $c, d \in L^{1}(0, T)$, we write $c \prec d$ if $c(t) \leq d(t)$ for a.e. $t \in[0, T]$ and $c(t)<d(t)$ on a set of positive measure.

Lyapunov's result is remarkable, among others, for two main reasons: first, one can check (1.3) directly from the equation (1.1) and second, it is optimal in the following sense ([31]): for any $\varepsilon \in \mathbb{R}^{+}$, there are unstable differential equations (1.1) with $a$ satisfying (1.2), for which

$$
0 \prec a, \int_{0}^{T} a(t) d t \leq \frac{4}{T}+\varepsilon
$$

Condition (1.3) has been generalized in several ways ([2], 25]). More recently the authors provide in 38 optimal stability criteria by using $L^{p}$ norms of $a^{+}, 1 \leq p \leq \infty$.

The parametric equation

$$
u^{\prime \prime}(t)+(\mu+a(t)) u(t)=0, \mu \in \mathbb{R},
$$

plays a fundamental role in the study of the stability properties of the Hill equation (1.1). In fact, if $\lambda_{i}(a), i \in \mathbb{N} \cup\{0\}$ and $\tilde{\lambda}_{i}(a), i \in \mathbb{N}$, denote, respectively, the eigenvalues of (1.4) for the periodic $\left(u(0)-u(T)=u^{\prime}(0)-\right.$ $\left.u^{\prime}(T)=0\right)$ and antiperiodic $\left(u(0)+u(T)=u^{\prime}(0)+u^{\prime}(T)=0\right)$ problem, 
then Lyapunov and Haupt proved (see ([17, 21] and [31] for historical and mathematical details) that

$$
\lambda_{0}(a)<\tilde{\lambda}_{1}(a) \leq \tilde{\lambda}_{2}(a)<\lambda_{1}(a) \leq \lambda_{2}(a)<\tilde{\lambda}_{3}(a) \leq \tilde{\lambda}_{4}(a)<\lambda_{3}(a) \leq \ldots
$$

and that equation (1.4) is stable if

$$
\mu \in\left(\lambda_{2 n}(a), \tilde{\lambda}_{2 n+1}(a)\right) \cup\left(\tilde{\lambda}_{2 n+2}(a), \lambda_{2 n+1}(a)\right)
$$

for some $n \in \mathbb{N} \cup\{0\}$. As a consequence, if either

$$
\lambda_{2 n}(a)<0<\tilde{\lambda}_{2 n+1}(a)
$$

or

$$
\tilde{\lambda}_{2 n+2}(a)<0<\lambda_{2 n+1}(a)
$$

then (1.1) is stable. In this case, we say that $\mu=0$ belongs to the $n^{\text {th }}$ stability zone of (1.4). In particular, the Lyapunov's conditions (1.3) imply $\lambda_{0}(a)<0<\tilde{\lambda}_{1}(a)$.

Except in very special cases, it is not an easy task to obtain some information on the sign of the previous eigenvalues. One possibility is to use the variational characterization, but in general it is a difficult question, especially at higher eigenvalues.

It is at this point where the so called "Lyapunov inequalities" can play an important role. In fact, the problem of the determination of sufficient conditions to have the desired properties (1.7) or (1.8), is intimately connected with the problem of finding the so called best Lyapunov constant for certain linear and homogeneous boundary value problems (Theorem 2.1 and Theorem 2.2 in the second section). Illustrate this with an example for periodic boundary conditions.

Let us consider the problem

$$
u^{\prime \prime}(t)+a(t) u(t)=0, t \in(0, T), u(0)-u(T)=u^{\prime}(0)-u^{\prime}(T)=0
$$

where $a \in L_{T}(\mathbb{R}, \mathbb{R})$. We denote

$$
\lambda_{0}(0)=\lambda_{0}=0, \lambda_{2 n-1}(0)=\lambda_{2 n}(0)=\lambda_{2 n-1}=\lambda_{2 n}=(2 n)^{2} \pi^{2} / T^{2}, n \in \mathbb{N} .
$$

Then, if $n \in \mathbb{N}$ is fixed, we introduce the set $\Lambda_{n}$ as

(1.10) $\Lambda_{n}=\left\{a \in L_{T}(\mathbb{R}, \mathbb{R}): \lambda_{2 n-1} \prec a\right.$ and (1.9) has nontrivial solutions $\}$

and define the Lyapunov constant (at higher eigenvalues) as

$$
\gamma_{1, n}=\inf _{a \in \Lambda_{n}}\|a\|_{L^{1}(0, T)}
$$

Then if a given function $a \in L_{T}(\mathbb{R}, \mathbb{R})$ satisfies

$$
\lambda_{2 n-1} \prec a,\|a\|_{L^{1}(0, T)}<\gamma_{1, n}
$$

we have

$$
\lambda_{2 n}(a)<0<\lambda_{2 n+1}(a) .
$$


In fact, since $\lambda_{2 n-1} \prec a$, by using the variational characterization of the periodic eigenvalues, we trivially have $\lambda_{2 n}(a)<\lambda_{2 n}\left(\lambda_{2 n-1}\right)=0$. To prove that $\lambda_{2 n+1}(a)>0$ we can use the following continuation method (which, to the best of our knowledge, has been introduced by the authors in [13]): let us define the continuous function $g:[0,1] \rightarrow \mathbb{R}$ by

$$
g(\varepsilon)=\lambda_{2 n+1}\left(a_{\varepsilon}(\cdot)\right)
$$

where $a_{\varepsilon}(x)=\lambda_{2 n-1}+\varepsilon\left(a(\cdot)-\lambda_{2 n-1}\right)$. Then $g(0)=\lambda_{2 n+1}\left(\lambda_{2 n-1}\right)=$ $\lambda_{2 n+1}-\lambda_{2 n-1}>0$. Moreover, for each $\varepsilon \in(0,1)$, the function $a_{\varepsilon}$ satisfies: $a_{\varepsilon} \in L_{T}(\mathbb{R}, \mathbb{R}), \lambda_{2 n-1} \prec a_{\varepsilon}$ and $\left\|a_{\varepsilon}\right\|_{L^{1}(0, T)}<\gamma_{1, n}$. Therefore, taking into account the definition of $\gamma_{1, n}$, the real number zero is not an eigenvalue of the eigenvalue problem

$$
u^{\prime \prime}(t)+\left(\mu+a_{\varepsilon}(t)\right) u(t)=0, t \in(0, T), \quad u(0)-u(T)=u^{\prime}(0)-u^{\prime}(T)=0 .
$$

In particular, $\lambda_{2 n+1}\left(a_{\varepsilon}(\cdot)\right) \neq 0$, i.e. $g(\varepsilon) \neq 0, \forall \varepsilon \in(0,1]$ and consequently, we obtain $g(1)=\lambda_{2 n+1}(a)>0$.

These arguments can be used not only for $L_{1}$-norms but also for $L_{p^{-}}$ norms with $1 \leq p \leq \infty$ and for different boundary conditions such as antiperiodic, Dirichlet, Neumann, etc. As we show in Theorem 2.1 and Theorem 2.2, we use a common procedure to study this kind of problems and also (see the previous commentaries to Theorem 2.1) for obtaining possible applications, which include not only stability theory, but also resonant (linear or nonlinear) boundary value problems (see Theorem 4.2 and Theorem 5.3). Of course, each boundary value problem has its peculiarities and, moreover, the cases $p=1, p=\infty$ and $1<p<\infty$ are generally different from a qualitative point of view, specially in the problems of the calculus of variations associated to them (see Theorem 3.1). These problems are, among other things, of interest in crystallography ([18], [19]).

One last general comment before describing the contents of the sections of the paper: this type of question can be seen as an optimal control problem and some authors have used the Pontryagin maximum principle in its study ([20, 23], 29], 37]). For example, for the periodic problem, the admissible control set is $\Lambda_{n}$ defined in (1.10) and the functional that we want to minimize is $\Phi: \Lambda_{n} \rightarrow \mathbb{R}$, defined as $\Phi(a)=\int_{0}^{T} a(t) d t, \forall a \in \Lambda_{n}$. However, we caution that the condition

$$
\text { (1.9) has nontrivial solutions }
$$

is difficult to handle from a mathematical point of view and this is the main difficulty of the problem. We will overcome these difficulties by using different methods of classical analysis such as a modified version of Sturm separation theorem, constrained or unconstrained minimization problems of the calculus of variations (depending on the boundary value problem that we are considering), Euler equation, Lagrange multiplier theorem, continuation methods, Schauder fixed point theorem, etc. 
In the second section of this paper we consider scalar ordinary differential equations. First we study the best $L_{1}$ Lyapunov constant at higher eigenvalues for the periodic and the antiperiodic boundary value problem. Then, we apply these results to obtain some new sufficient conditions on the $n^{\text {th }}$ stability zone of the Hill equation (1.1).

The third section is dedicated to systems of equations like

$$
u^{\prime \prime}(t)+P(t) u(t)=0, t \in \mathbb{R},
$$

where the matrix function $P(\cdot)$ is $T$-periodic. Despite its undoubted interest (see references [17], 21], 25], [31] to get an idea of the many applications of this kind of systems), there are not many studies on this subject. We must remark that the results we show in the scalar case are mainly based on an exact knowledge about the number and distribution of the zeros of the corresponding solutions, but to the best of our knowledge, for systems of equations like (1.13), we do not know similar results to those given in Theorem 2.1 and Theorem 2.2 .

To extend the obtained results in the scalar case to systems of equations, a key point is the characterization of the best $L_{p}$ Lyapunov constant for the scalar given problem, as a minimum of some especial minimization problems defined in appropriate subsets $X_{p}, 1 \leq p \leq \infty$, of the Sobolev space $H^{1}(0, T)$. For the Dirichlet problem this was done by Talenti ([36]) and for the Neumann and periodic problem this was done by the authors in [6] and [14] (see also [18] for a different treatment and motivation of the problem). Since 0 is the first eigenvalue for Neumann and periodic boundary problems, it is necessary to impose an additional restriction to the definition of the spaces $X_{p}, 1 \leq p \leq \infty$ in the case of Neumann and periodic conditions (see Remark 8 below), and therefore we will have constrained minimization problems. This is not necessary in the case of Dirichlet or antiperiodic boundary ones where we will find unconstrained minimization problems.

In regard to stability properties of (1.13), which will be considered also in Section 3, the results proved by Krein in 25] show that the problem is closely related to Lyapunov inequalities. In fact, under appropriate hypotheses on the function matrix $P(\cdot)$ (see Subsection 3.2), the stability properties of (1.13) strongly depend on the fact that the smallest positive eigenvalue of the antiperiodic eigenvalue problem

$$
u^{\prime \prime}(t)+\lambda P(t) u(t)=0, t \in \mathbb{R}, u(0)+u(T)=u^{\prime}(0)+u^{\prime}(T)=0
$$

be greater than one. We establish some new conditions to get this last property.

Section 4 will be devoted to the study of $L_{p}$ Lyapunov inequalities and some applications to nonlinear resonant problems for scalar partial differential equations. More precisely, we consider the linear problem with Neumann 
boundary conditions

$$
\left.\begin{array}{cl}
\Delta u(x)+a(x) u(x)=0 & x \in \Omega \\
\frac{\partial u}{\partial n}(x)=0 & x \in \partial \Omega
\end{array}\right\}
$$

where $\Omega \subset \mathbb{R}^{N}(N \geq 2)$ is a bounded and regular domain and $\frac{\partial}{\partial n}$ is the outer normal derivative on $\partial \Omega$.

According to our opinion, the work by the authors [8] and [9] showed for the first time significant differences from the ordinary case. The most important is, without doubt, the following fact: if $N=2$, there is $L_{p}$ Lyapunov inequality if and only if $1<p \leq \infty$ and if $N \geq 3$, there is $L_{p}$ Lyapunov inequality if and only if $N / 2 \leq p \leq \infty$. In particular, we must remark that there is no $L_{1}$ Lyapunov inequality in the PDE case and that the best constant is attained if $N / 2<p \leq \infty$.

A pioneering work for the case of Dirichlet boundary conditions $u(x)=$ $0, x \in \partial \Omega$, was undoubtedly the Talenti article [36, where the author relates this kind of inequalities (called Sobolev Inequalities in this and in other many papers in PDE), with the classic isoperimetric problem for subsets of Euclidean space. Talenti made a detailed study of the case where $\Omega$ is substituted by the whole space $\mathbb{R}^{N}, N \geq 3$ and $p=N / 2$ (the critical value), including the study of some additional symmetric properties for those functions where the considered infimum is attained.

By using our methods, in the case of Dirichlet conditions it is possible to obtain analogous results and if $N / 2<p<\infty$, the Lyapunov constant for the Dirichlet problem, $\beta_{p}^{D}$, can be characterized variationally as

$$
\beta_{p}^{D}=\inf _{u \in H_{0}^{1}(\Omega) \backslash\{0\}} \frac{\int_{\Omega}|\nabla u|^{2}}{\left(\int_{\Omega}|u|^{\frac{2 p}{p-1}}\right)^{\frac{p-1}{p}}}
$$

If $\Omega$ is, moreover, a radial domain, previous minimization problem is related to a more general one which involves Rayleigh quotient

$$
\frac{\int_{\Omega}|\nabla u|^{2}}{\left(\int_{\Omega} \rho(x)|u|^{\frac{2 p}{p-1}}\right)^{\frac{p-1}{p}}}
$$

where $\rho \in L^{q}(\Omega), q=N(p-1) /(2 p-N)$, is a positive function. This has been used in the study of the existence of nonsymmetric ground states of symmetric problems for nonlinear PDE's (see [4, 5] and [35]).

In Section 4, first we treat the scalar linear problem (1.15). Then we apply the linear study to nonlinear resonant problems by using the Schauder fixed point theorem (see Theorem 4.2). Obviously, similar results may be proved 
for nonlinear and resonant ordinary differential equations for each value $1 \leq p \leq \infty$.

In Section 5, we deal with elliptic systems of the form

$$
\Delta u(x)+A(x) u(x)=0, x \in \Omega, \quad \frac{\partial u(x)}{\partial n}=0, x \in \partial \Omega,
$$

where $A(\cdot)$ is a real $n \times n$ symmetric and continuous matrix satisfying some additional restrictions. To our knowledge, there are no previous work on $L_{p}$ Lyapunov inequalities for elliptic systems like (1.16) if $p \neq \infty$ (see [1] and [24, Section 5, for the case $p=\infty$ ).

As in the ordinary case, to extend to systems of equations the obtained results in the scalar case, a key point is the characterization of the best $L_{p}$ Lyapunov constant for the scalar given problem, as a minimum of some especial minimization problems defined in appropriate subsets $X_{p}, N / 2<$ $p \leq \infty$, of the Sobolev space $H^{1}(\Omega)$.

By combining the linear study with Schauder fixed point theorem, we show some applications to nonlinear resonant problems. In particular, and for Neumann boundary conditions, we obtain a generalization for systems of equations of the main result given in [34] where the authors treated the scalar case and where they use in the proof the duality method of Clarke and Ekeland.

Finally, we present some remarks on other interesting aspects not treated here and also on some open problems.

\section{SCALAR ORDINARY DIFFERENTIAL EQUATIONS}

This section deals with $L_{1}$ Lyapunov inequality for the periodic and the antiperiodic boundary value problem at higher eigenvalues. As an application, we obtain some new stability properties on the Hill equation (1.1).

We explain in more detail what we mean by $L_{1}$ Lyapunov inequality at higher eigenvalues. Remember that $L_{T}(\mathbb{R}, \mathbb{R})$ denotes the set of $T$-periodic functions $a: \mathbb{R} \rightarrow \mathbb{R}$, such that $\left.a\right|_{[0, T]} \in L^{1}(0, T)$. Then, we will consider the periodic boundary value problem

$$
u^{\prime \prime}(t)+a(t) u(t)=0, t \in(0, T), u(0)-u(T)=u^{\prime}(0)-u^{\prime}(T)=0
$$

It is very well known ([17], [21], [22]) that the eigenvalues for

$$
u^{\prime \prime}(t)+(\lambda+a(t)) u(t)=0, t \in(0, T), u(0)-u(T)=u^{\prime}(0)-u^{\prime}(T)=0
$$

form a sequence $\lambda_{n}(a), n \in \mathbb{N} \cup\{0\}$, such that

$$
\lambda_{0}(a)<\lambda_{1}(a) \leq \lambda_{2}(a)<\ldots<\lambda_{2 n-1}(a) \leq \lambda_{2 n}(a)<\ldots
$$

with $\lambda_{0}(a)$ simple and such that if $\phi_{n}$ is the corresponding eigenfunction to $\lambda_{n}(a)$, then $\phi_{0}$ has no zeros in $[0, T]$ and $\phi_{2 n-1}$ and $\phi_{2 n}$ have exactly $2 n$ 
zeros in $[0, T)$. In particular, $\lambda_{0}=\lambda_{0}(0)=0, \lambda_{2 n-1}=\lambda_{2 n}=\lambda_{2 n-1}(0)=$ $\lambda_{2 n}(0)=(2 n)^{2} \pi^{2} / T^{2}, n \in \mathbb{N}$.

Similarly, we will consider the antiperiodic boundary value problem

$$
u^{\prime \prime}(t)+a(t) u(t)=0, t \in(0, T), u(0)+u(T)=u^{\prime}(0)+u^{\prime}(T)=0
$$

where $a \in L_{T}(\mathbb{R}, \mathbb{R})$. It is also very well known ([17, [21], [22]) that the eigenvalues for

$$
u^{\prime \prime}(t)+(\tilde{\lambda}+a(t)) u(t)=0, t \in(0, T), u(0)+u(T)=u^{\prime}(0)+u^{\prime}(T)=0
$$

form a sequence $\tilde{\lambda}_{n}(a), n \in \mathbb{N}$, such that

$$
\tilde{\lambda}_{1}(a) \leq \tilde{\lambda}_{2}(a)<\ldots<\tilde{\lambda}_{2 n-1}(a) \leq \tilde{\lambda}_{2 n}(a)<\ldots
$$

and if $\tilde{\phi}_{n}$ is the corresponding eigenfunction to $\tilde{\lambda}_{n}(a)$, then $\tilde{\phi}_{2 n-1}$ and $\tilde{\phi}_{2 n}$ have exactly $2 n-1$ zeros in $[0, T)$. In particular, $\tilde{\lambda}_{2 n-1}(0)=\tilde{\lambda}_{2 n}(0)=$ $(2 n-1)^{2} \pi^{2} / T^{2}, n \in \mathbb{N}$. We will denote $\tilde{\lambda}_{i}=\tilde{\lambda}_{i}(0), \forall i \in \mathbb{N}$.

It is clear that if the function $a(\cdot)$ is not a constant, it is not possible, in general, to calculate explicitly the mentioned eigenvalues. Specifically, it is not easy to establish that the real number 0 is not an eigenvalue of (2.2) (or (2.5)). Perhaps, for the periodic problem (2.1), the following result is intuitive and understandable: if there exists some $n \in \mathbb{N}$ such that $\lambda_{2 n-1} \prec$ $a \prec \lambda_{2 n+1}$ (or $\lambda_{0} \prec a \prec \lambda_{1}$ ), then the unique solution of (2.1) is the trivial one, i.e., 0 is not an eigenvalue of (2.2) (see [27]). A similar result is true for the antiperiodic problem.

The previous criterion may be seen as a $L_{\infty}-L_{\infty}$ criterion in the sense that the function $a$ is bounded, both above and below, by consecutive different eigenvalues $\lambda_{2 n-1}, \lambda_{2 n+1}$. It allows a weak interaction between the function $a$ and the corresponding spectrum. Our next purpose is to show a $L_{\infty}-$ $L_{1}$ criterion. It will allow the function $a$ to cross an arbitrary number of eigenvalues as long as certain $L_{1}$-norms are controlled.

More specifically, if $n \in \mathbb{N}$ is fixed, we introduce for the periodic problem, the set $\Lambda_{n}$ as

$$
\Lambda_{n}=\left\{a \in L_{T}(\mathbb{R}, \mathbb{R}): \lambda_{2 n-1} \prec a \text { and (2.1) has nontrivial solutions }\right\}
$$

Our main interest is in having a broader knowledge as possible about the constant

$$
\gamma_{1, n}=\inf _{a \in \Lambda_{n}}\|a\|_{L^{1}(0, T)}
$$

Analogously, if $n \in \mathbb{N}$ is fixed, we can introduce for the antiperiodic problem the set $\tilde{\Lambda}_{n}$ as

$$
\tilde{\Lambda}_{n}=\left\{a \in L_{T}(\mathbb{R}, \mathbb{R}): \tilde{\lambda}_{2 n-1} \prec a \text { and (2.4) has nontrivial solutions }\right\}
$$


and, as for the periodic problem, our main interest is in having a broader knowledge as possible about the constant

$$
\tilde{\gamma}_{1, n} \equiv \inf _{a \in \tilde{\Lambda}_{n}}\|a\|_{L^{1}(0, T)}
$$

The above ideas clarify the meaning of the expression " $L_{1}$ Lyapunov inequalities at higher eigenvalues".

Additionally, we prove some qualitative properties on the sign of the eigenvalues $\lambda_{2 n}(a), \lambda_{2 n+1}(a), \tilde{\lambda}_{2 n}(a), \tilde{\lambda}_{2 n+1}(a)$, which will be very important in the applications to resonant problems and in the study of stability properties of linear periodic equations. To prove these qualitative properties, we use a curious continuation method applied to an especial continuous function which does not change its sign in its domain (an interval).

2.1. Lyapunov constants for the periodic and the antiperiodic boundary value problem. First, we deal with the constant $\gamma_{1, n}$ defined in (2.8). Note that if $a \in \Lambda_{n}$, and $u$ is any nontrivial solution of (2.1), then $u$ is not a constant function and $u$ must have a zero in the interval $[0, T]$. If $r \in[0, T]$ is such that $u(r)=0$, the periodic and nontrivial function $v(t)=u(r+t)$ satisfies $v(0)=v(T)=0, v^{\prime \prime}(t)+a(r+t) v(t)=0, t \in(0, T)$ and $\|a(r+\cdot)\|_{L^{1}(0, T)}=\|a(\cdot)\|_{L^{1}(0, T)}$. This observation does possible to consider in our study only those situations where $u$ is such that $u(0)=u(T)=0$. Since $a \in \Lambda_{n}, n \in \mathbb{N}$, it is clear that between two consecutive zeros of the function $u$ there must exists a zero of the function $u^{\prime}$ and between two consecutive zeros of the function $u^{\prime}$ there must exists a zero of the function $u$.

In the next theorem, we provide a comprehensive review of the control problem which we have described in (2.7) and (2.8) and we show some qualitative properties of the eigenvalues $\lambda_{2 n}(a), \lambda_{2 n+1}(a)$ associated to those functions $a \in L_{T}(\mathbb{R}, \mathbb{R})$ such that $\Phi(a)=\int_{0}^{T} a(t) d t \leq \gamma_{1, n}$. We consider these qualitative properties (and similar ones which will be obtained for the antiperiodic boundary value problem) interesting in themselves.

In the proof, the following fundamental ideas are used:

(1) A careful analysis on the number and distribution of zeros of nontrivial solutions $u$ of the equation (2.1) (or the equation (2.4) ) and their first derivatives $u^{\prime}$, including an optimal estimation about the corresponding distances between the zeros of $u$ and $u^{\prime}$. To this respect, we compare our problem with other one with mixed boundary conditions. This allows us to obtain a more precise information than that obtained when the classical Sturm separation theorem is used, where the problem is compared with the case of Dirichlet boundary conditions ([22] ) and therefore, the obtained information is only on the zeros of $u$. 
(2) The previous step will motivate the study of the following minimization problem $\left(H^{1}(a, b)\right.$ denotes the usual Sobolev space):

Let $H=\left\{u \in H^{1}(a, b): u(a)=0, u(b) \neq 0\right\}$ and $J: H \rightarrow \mathbb{R}$ be defined by

$$
J(u)=\frac{\int_{a}^{b} u^{\prime 2}-M \int_{a}^{b} u^{2}}{u^{2}(b)}
$$

where $a<b$ and $0<M \leq \frac{\pi^{2}}{4(b-a)^{2}}$, the first (or principal) eigenvalue of the eigenvalue problem with mixed boundary conditions

$$
u^{\prime \prime}(t)+\mu u(t)=0, t \in(a, b), u(a)=0, u^{\prime}(b)=0 .
$$

Then, with the help of classical and standard methods of the calculus of variations (existence of minimizing sequences and the study of the Euler-Lagrange equation), we obtain that $c \equiv \inf _{u \in H} J(u)$, is attained and

$$
c=M^{1 / 2} \cot \left(M^{1 / 2}(b-a)\right)
$$

Moreover, if $u \in H$, then $J(u)=c \Longleftrightarrow u(t)=k \sin \left(M^{1 / 2}(t-a)\right)$ for some non zero constant $k$.

(3) The final step is the study of a simple minimization problem which is given by a finite sum of functionals of the previous type. More precisely, given any $r \in \mathbb{N}$ and $S \in \mathbb{R}^{+}$satisfying $r \pi>2 S$, let

$$
Z=\left\{z=\left(z_{0}, z_{1}, \ldots, z_{r-1}\right) \in(0, \pi / 2]^{r}: \sum_{i=0}^{r-1} z_{i}=S\right\}
$$

If $F: Z \rightarrow \mathbb{R}$ is defined by

$$
F(z)=\sum_{i=0}^{r-1} \cot z_{i}
$$

we will show that $\inf _{z \in Z} F(z)$ is attained and its value is $r \cot \frac{S}{r}$. Moreover, $z \in Z$ is a minimizer if and only if $z_{i}=\frac{S}{r}, \forall 0 \leq i \leq r-1$. To obtain these conclusions, we basically use Lagrange multiplier theorem.

Theorem 2.1. Let $n \in \mathbb{N}$ and $a \in \Lambda_{n}$ be given and $u$ any nontrivial solution of (2.1) such that $u(0)=u(T)=0$. If the zeros of $u$ in $[0, T]$ are denoted by $0=t_{0}<t_{2}<\ldots<t_{2 m}=T$ and the zeros of $u^{\prime}$ in $(0, T)$ are denoted by $t_{1}<t_{3}<\ldots<t_{2 m-1}$, then:

(1) $t_{i+1}-t_{i} \leq \frac{T}{4 n}, \forall i: 0 \leq i \leq 2 m-1$. Moreover, at least one of these inequalities is strict.

(2) $m$ is an even number and $m \geq 2(n+1)$. Any even value $m \geq 2(n+1)$ is possible. 
(3)

$\gamma_{1, n}$ is not attained and

$$
\gamma_{1, n}=T \lambda_{2 n-1}+\frac{8 \pi n(n+1)}{T} \cot \frac{n \pi}{2(n+1)}
$$

(4) If $a \in L_{T}(\mathbb{R}, \mathbb{R})$ satisfies

$$
\lambda_{2 n-1} \prec a,\|a\|_{L^{1}(0, T)} \leq \gamma_{1, n}
$$

then

$$
\lambda_{2 n}(a)<0<\lambda_{2 n+1}(a)
$$

Proof. We only summary the main ideas (see [12, Theorem 2.1 and [13], Theorem 2.1 for the details).

First, let $i, 0 \leq i \leq 2 m-1$, be given. Then, function $u$ satisfies either the problem

$$
u^{\prime \prime}(t)+a(t) u(t)=0, t \in\left(t_{i}, t_{i+1}\right), \quad u\left(t_{i}\right)=0, u^{\prime}\left(t_{i+1}\right)=0,
$$

or the problem

$$
u^{\prime \prime}(t)+a(t) u(t)=0, t \in\left(t_{i}, t_{i+1}\right), \quad u^{\prime}\left(t_{i}\right)=0, u\left(t_{i+1}\right)=0 .
$$

Let us assume the first case and let us denote by $\mu_{1}^{i}=\frac{\pi^{2}}{4\left(t_{i+1}-t_{i}\right)^{2}}$ and $\varphi_{1}^{i}(t)=$ $\sin \frac{\pi\left(t-t_{i}\right)}{2\left(t_{i+1}-t_{i}\right)}$, respectively, the principal eigenvalue and eigenfunction of the eigenvalue problem

$$
v^{\prime \prime}(t)+\mu v(t)=0, t \in\left(t_{i}, t_{i+1}\right), v\left(t_{i}\right)=0, v^{\prime}\left(t_{i+1}\right)=0 .
$$

Choosing $\varphi_{1}^{i}$ as test function in the weak formulation of (2.18) and $u$ as test function in the weak formulation of (2.20) for $\mu=\mu_{1}^{i}$ and $v=\varphi_{1}^{i}$, we obtain

$$
\int_{t_{i}}^{t_{i+1}}\left(a(t)-\mu_{1}^{i}\right) u(t) \varphi_{1}^{i}(t) d t=0 .
$$

This last relation and the hypothesis $\lambda_{2 n-1} \prec a$, prove the first part of the theorem and also the relation $m \geq 2 n+1$. Since we are considering the periodic problem, $m$ must be an even number and therefore, $m \geq 2(n+1)$. Also, note that for any given even and natural number $q \geq 2(n+1)$, function $b(x) \equiv \lambda_{q}$ belongs to $\Lambda_{n}$ and for function $v(x)=\sin \frac{q \pi x}{T}$, we have $m=q$. In this way, we have proved the first two parts of the theorem.

Continuing with the proof, if $i$, with $0 \leq i \leq 2 m-1$ is given and $u$ satisfies (2.18), then

$$
\begin{gathered}
\int_{t_{i}}^{t_{i+1}} u^{\prime 2}(t)=\int_{t_{i}}^{t_{i+1}} a(t) u^{2}(t)= \\
\int_{t_{i}}^{t_{i+1}}\left(a(t)-\lambda_{2 n-1}\right) u^{2}(t)+\int_{t_{i}}^{t_{i+1}} \lambda_{2 n-1} u^{2}(t)
\end{gathered}
$$

Therefore,

$$
\int_{t_{i}}^{t_{i+1}} u^{\prime 2}(t)-\lambda_{2 n-1} \int_{t_{i}}^{t_{i+1}} u^{2}(t) \leq\left\|a-\lambda_{2 n-1}\right\|_{L^{1}\left(t_{i}, t_{i+1}\right)}\left\|u^{2}\right\|_{L^{\infty}\left(t_{i}, t_{i+1}\right)}
$$


Since $u^{\prime}$ has no zeros in the interval $\left(t_{i}, t_{i+1}\right)$ and $u\left(t_{i}\right)=0$, we have $\left\|u^{2}\right\|_{L^{\infty}\left(t_{i}, t_{i+1}\right)}=u^{2}\left(t_{i+1}\right)$. This proves (see (2.13) $)$

$\left\|a-\lambda_{2 n-1}\right\|_{L^{1}\left(t_{i}, t_{i+1}\right)} \geq \frac{\int_{t_{i}}^{t_{i+1}} u^{\prime 2}-\lambda_{2 n-1} \int_{t_{i}}^{t_{i+1}} u^{2}}{u^{2}\left(t_{i+1}\right)} \geq \frac{2 n \pi}{T} \cot \left(\frac{2 n \pi}{T}\left(t_{i+1}-t_{i}\right)\right)$.

In particular, this implies

$$
\left\|a-\lambda_{2 n-1}\right\|_{L^{1}(0, T)} \geq \frac{2 n \pi}{T} \sum_{i=0}^{2 m-1} \cot \left(\frac{2 n \pi}{T}\left(t_{i+1}-t_{i}\right)\right)
$$

The right-hand side of (2.23) attains its minimum if and only if $t_{i+1}-t_{i}=$ $\frac{T}{2 m}, 0 \leq i \leq 2 m-1$ (see Lemma 2.5 in [12]) so that

$$
\gamma_{1, n} \geq T \lambda_{2 n-1}+\frac{4 n \pi}{T} m \cot \frac{n \pi}{m}
$$

Taking into account that the function $m \cot \frac{n \pi}{m}$ is strictly increasing with respect to $m$, and that $m \geq 2(n+1)$, we deduce

$$
\gamma_{1, n} \geq T \lambda_{2 n-1}+\frac{8 \pi n(n+1)}{T} \cot \frac{n \pi}{2(n+1)} .
$$

At this point, an appropriate minimizing sequence for $\gamma_{1, n}$ may be constructed. The details are very technical and they do not contribute anything important. On the other hand, a careful observation of the optimality properties of the different previos inequalities, do possible to prove that $\gamma_{1, n}$ is not attained (see Lemma 2.3 and Lemma 2.4 in [13]).

For the last part of the theorem, let us assume that the function $a$ satisfies (2.16) . Then, since $\lambda_{2 n-1} \prec a$, we trivially have $\lambda_{2 n}(a)<\lambda_{2 n}\left(\lambda_{2 n-1}\right)=0$. To prove that $\lambda_{2 n+1}(a)>0$ we use a continuation method: let us define the continuous function $g:[0,1] \rightarrow \mathbb{R}$ by

$$
g(\varepsilon)=\lambda_{2 n+1}\left(a_{\varepsilon}(\cdot)\right)
$$

where $a_{\varepsilon}(\cdot)=\lambda_{2 n-1}+\varepsilon\left(a(\cdot)-\lambda_{2 n-1}\right)$. Then $g(0)=\lambda_{2 n+1}\left(\lambda_{2 n-1}\right)=\lambda_{2 n+1}-$ $\lambda_{2 n-1}>0$. Moreover, $g(\varepsilon) \neq 0, \forall \varepsilon \in(0,1]$. In fact, we deduce from the previous parts of the theorem that the number 0 is not an eigenvalue of the function $a_{\varepsilon}$ for the periodic boundary conditions. As a consequence, $\lambda_{2 n+1}(a)=g(1)>0$ and the theorem is proved.

Remark 1. We can obtain similar results if, in the definition of the set $\Lambda_{n}$ in (2.7), we consider $n \in \mathbb{R}^{+}$instead of $n \in \mathbb{N}$. This may be important in order to obtain new stability results for periodic linear equations, as it is shown in Theorem 2.3 below. Only some minor changes are necessary. From this point of view, if we consider $\gamma_{1, n}$ as a function of $n \in(0,+\infty)$, then $\lim _{n \rightarrow 0^{+}} \gamma_{1, n}=\frac{16}{T}$, the constant of the classical $L^{1}$ Lyapunov inequality for the periodic problem at the first eigenvalue, which was obtained in [23] by using methods of optimal control theory. 
In the case $n=0$, we can use similar reasonings taking $(2.26)$

$\Lambda_{0}=\left\{a \in L_{T}(\mathbb{R}, \mathbb{R}) \backslash\{0\}: 0 \leq \int_{0}^{T} a(t) d t\right.$ and (2.1) has nontrivial solutions $\}$

In this case $m \geq 2$ and any even value $m \geq 2$ is possible. Consequently

$$
\gamma_{1,0}=\inf _{a \in \Lambda_{0}}\left\|a^{+}\right\|_{L^{1}(0, T)}=\frac{16}{T}
$$

Let us remark that the restriction

$$
a \in L_{T}(\mathbb{R}, \mathbb{R}) \backslash\{0\}: \lambda_{0}=0 \leq \int_{0}^{T} a(t) d t
$$

is more general that the restriction $\lambda_{0} \prec a$

(As a consequence, it is possible to obtain a $L_{1}-L_{1}$ Lyapunov inequality at the first eigenvalue).

Remark 2. The case where $T=2 \pi$ and function $a$ satisfies the condition $A \leq a(t) \leq B$, a.e. in $(0,2 \pi)$ where $k^{2}<A<(k+1)^{2}<B$ for some $k \in \mathbb{N} \cup\{0\}$, has been considered in [37, where the authors also use optimal control theory methods.

Remark 3. We can use our methods to do an analogous study for other boundary value problems (see the next theorem for antiperiodic boundary conditions). Consider that, after seeing the above reasonings, the key point is to have an optimal knowledge about the number and distribution of zeros of the functions $u$ and $u^{\prime}$, moreover of knowing the best value of the constant $m$ (given in the previous theorem). For example, for the periodic problem the optimal value of $m$ is $2(n+1)$ while for the antiperiodic problem the optimal value is $m=2 n+1$. See, for example, [12 for the case of Neumann, Dirichlet or mixed boundary conditions at higher eigenvalues.

Thinking in the next subsection, we finish this part with a similar theorem to Theorem 2.1 for the antiperiodic problem.

Theorem 2.2. Let $n \in \mathbb{N}$ and $a \in \tilde{\Lambda}_{n}$ be given and $u$ any nontrivial solution of (2.4) such that $u(0)=u(T)=0$. If the zeros of $u$ in $[0, T]$ are denoted by $0=t_{0}<t_{2}<\ldots<t_{2 m}=T$ and the zeros of $u^{\prime}$ in $(0, T)$ are denoted by $t_{1}<t_{3}<\ldots<t_{2 m-1}$, then:

(1) $t_{i+1}-t_{i} \leq \frac{T}{2(2 n-1)}, \forall i: 0 \leq i \leq 2 m-1$. Moreover, at least one of these inequalities is strict.

(2) $m$ is an odd number and $m \geq 2 n+1$. Any odd value $m \geq 2 n+1$ is possible. 
(3) $\tilde{\gamma}_{1, n}$ is not attained and

$$
\tilde{\gamma}_{1, n} \equiv \inf _{a \in \tilde{\Lambda}_{n}}\|a\|_{L^{1}(0, T)}=T \tilde{\lambda}_{2 n-1}+\frac{2 \pi(2 n-1)(2 n+1)}{T} \cot \frac{(2 n-1) \pi}{2(2 n+1)}
$$

(4) If $a \in L_{T}(\mathbb{R}, \mathbb{R})$ satisfies

$$
\tilde{\lambda}_{2 n-1} \prec a,\|a\|_{L^{1}(0, T)} \leq \tilde{\gamma}_{1, n},
$$

then

$$
\tilde{\lambda}_{2 n}(a)<0<\tilde{\lambda}_{2 n+1}(a)
$$

Remark 4. A similar theorem to the previous one may be proved if $a \in \tilde{\Lambda}_{0}$ where

$$
\tilde{\Lambda}_{0}=\left\{a \in L_{T}(\mathbb{R}, \mathbb{R}): \text { (2.4) has nontrivial solutions }\right\}
$$

In this case $m \geq 1$ and any even value $m \geq 1$ is possible. Consequently

$$
\tilde{\gamma}_{1,0}=\inf _{a \in \tilde{\Lambda}_{0}}\left\|a^{+}\right\|_{L^{1}(0, T)}=\frac{4}{T}
$$

Let us remark that the restriction $0 \prec a$ which is natural for the periodic problem (2.1), is not necessary in this case. This additional restriction is necessary in the Neumann problem (see Remark 4 in [6]), but not in the case of Dirichlet or mixed boundary conditions.

2.2. Stability of linear periodic equations. In this part of the paper, we deal with some stability properties of the Hill's equation

$$
u^{\prime \prime}(t)+a(t) u(t)=0, a \in L_{T}(\mathbb{R}, \mathbb{R}) .
$$

As we said in the Introduction, it is convenient to introduce the parametric equation

$$
u^{\prime \prime}(t)+(\mu+a(t)) u(t)=0, a \in L_{T}(\mathbb{R}, \mathbb{R}), \mu \in \mathbb{R} .
$$

If $\lambda_{i}(a), i \in \mathbb{N} \cup\{0\}$ and $\tilde{\lambda}_{i}(a), i \in \mathbb{N}$, denote, respectively the eigenvalues of (2.33) for the periodic and antiperiodic problem, then it is known ([17], [21]) that

$$
\lambda_{0}(a)<\tilde{\lambda}_{1}(a) \leq \tilde{\lambda}_{2}(a)<\lambda_{1}(a) \leq \lambda_{2}(a)<\tilde{\lambda}_{3}(a) \leq \tilde{\lambda}_{4}(a)<\lambda_{3}(a) \leq \ldots
$$

and that equation (2.34) is stable if

$$
\mu \in\left(\lambda_{2 n}(a), \tilde{\lambda}_{2 n+1}(a)\right) \cup\left(\tilde{\lambda}_{2 n+2}(a), \lambda_{2 n+1}(a)\right)
$$

for some $n \in \mathbb{N} \cup\{0\}$ and that equation (2.34) is unstable if

$$
\mu \in\left(-\infty, \lambda_{0}(a)\right] \cup\left(\lambda_{2 n+1}(a), \lambda_{2 n+2}(a)\right) \cup\left(\tilde{\lambda}_{2 n+1}(a), \tilde{\lambda}_{2 n+2}(a)\right)
$$

for some $n \in \mathbb{N} \cup\{0\}$. If $\mu=\lambda_{2 n+1}(a)$ or $\mu=\lambda_{2 n+2}(a)$, (2.34) is stable if and only if $\lambda_{2 n+1}(a)=\lambda_{2 n+2}(a)$ and, finally, if $\mu=\tilde{\lambda}_{2 n+1}(a)$ or $\mu=\tilde{\lambda}_{2 n+2}(a)$, (2.34) is stable if and only if $\tilde{\lambda}_{2 n+1}(a)=\tilde{\lambda}_{2 n+2}(a)$. 
As a consequence, if we establish conditions which assure either $\lambda_{2 n}(a)<$ $0<\tilde{\lambda}_{2 n+1}(a)$ or $\tilde{\lambda}_{2 n+2}(a)<0<\lambda_{2 n+1}(a)$ then (2.33) is stable. In this case, we say that $\mu=0$ belongs to the $n^{\text {th }}$ stability zone of (2.33).

In what follows in this subsection, we choose $T=\pi$, for simplicity. If one uses $L_{\infty}$ Lyapunov inequalities, the following result may be proved ( see [31, Chapter V, Theorem 5.5):

If $r$ and $s$ are given real numbers and

$$
r^{2} \leq a(t) \leq s^{2}
$$

then (2.33) is stable for all possible functions $a(\cdot)$ satisfying (2.38) if and only if the interval $\left(r^{2}, s^{2}\right)$ does not contain the square of an integer.

In particular, concerning to the first stability zone, (2.33) is stable if

$$
0 \leq a(t) \leq 1
$$

and for functions satisfying $0 \leq a(t)$, this result is optimal in the following sense: for any positive number $\varepsilon$ there is some function $a(t)$ with $a \in L_{T}(\mathbb{R}, \mathbb{R})$, satisfying $0 \leq a(t) \leq 1+\varepsilon$ and such that (2.33) is unstable.

The previous criterion on the stability of (2.33) may be considered as a $L_{\infty}-L_{\infty}$ criterion. We can exploit the results obtained in Theorem 2.1 and Theorem 2.2 to obtain new results on the stability properties of (2.33) of the type $L_{\infty}-L_{1}$. This is the purpose of the next theorem, where function $a$ can be cross an arbitrary number of squares of an integer, as long as its $L_{1}$-norm is properly controlled (see [13] for a more detailed proof).

Theorem 2.3. Let $a \in L_{\pi}(\mathbb{R}, \mathbb{R})$ satisfying

$$
\begin{gathered}
\exists p \in \mathbb{N}, \exists k \in\left[p^{2},(p+1)^{2}\right]: \\
k \leq a, \quad\|a\|_{L^{1}(0, \pi)} \leq k \pi+k^{1 / 2} 2(p+1) \cot \frac{k^{1 / 2} \pi}{2(p+1)}
\end{gathered}
$$

Then $\mu=0$ is in the $n^{\text {th }}$ stability zone of the Hill's equation 2.34).

Proof. It is clearly not restrictive (see Theorem 2.1) to assume that

$$
\begin{gathered}
\exists p \in \mathbb{N}, \exists k \in\left(p^{2},(p+1)^{2}\right): \\
k \prec a, \quad\|a\|_{L^{1}(0, \pi)} \leq k \pi+k^{1 / 2} 2(p+1) \cot \frac{k^{1 / 2} \pi}{2(p+1)}
\end{gathered}
$$

Let us suppose, for instance, that $p=2 n, n \in \mathbb{N}$. Then, $\lambda_{2 n}(a)<\lambda_{2 n}\left(\lambda_{2 n-1}\right)=$ 0 . On the other hand, since $k \prec a$, doing a similar reasoning to that in Theorem 2.1. but for the antiperiodic problem, we have that if $u$ is a nontrivial solution of (2.4) such that $u(0)=u(\pi)=0$, then $\left|x_{i+1}-x_{i}\right| \leq \frac{\pi}{2 k^{1 / 2}}$. This implies the relation $m>k^{1 / 2}$ in in Theorem 2.2. But since we are now considering the antiperiodic problem (2.4), $m$ must be an odd number. Also 
$p<k^{1 / 2}<p+1$, and as $p=2 n$, we deduce $m \geq 2 n+1$. Consequently,

$$
\|a-k\|_{L^{1}(0, \pi)} \geq k^{1 / 2} 2(2 n+1) \cot \left(\frac{k^{1 / 2} \pi}{2(2 n+1)}\right)
$$

(see (2.24)). Moreover, this last constant is not attained. As in Theorem 2.1, if $h:[0,1] \rightarrow \mathbb{R}$ is defined as por $h(\varepsilon)=\tilde{\lambda}_{2 n+1}(k+\varepsilon(a(\cdot)-k))$, we obtain $h(0)>0$ and (from (2.41) $), h(\varepsilon) \neq 0, \forall \varepsilon \in(0,1]$. Then, $h(1)=\tilde{\lambda}_{2 n+1}(a)>$ 0 . As a consequence, $\mu=0 \in\left(\lambda_{2 n}(a), \tilde{\lambda}_{2 n+1}(a)\right)$ and the theorem is proved. The proof is similar if $p$ is an odd number.

Remark 5. The case where $a(t)=\alpha+\beta \psi(t)$, with $\psi \in L_{\pi}(\mathbb{R}, \mathbb{R}), \int_{0}^{\pi} \psi(t) d t=$ 0 and $\int_{0}^{\pi}|\psi(t)| d t=1 / \pi$, was studied by Borg ([2]). Borg used the characteristic multipliers determined from Floquet's theory. He deduced stability criteria for (2.34) by using the two parameters $\alpha$ and $\beta$. For a concrete function $a$, this implies the use of the two quantities

$$
\int_{0}^{\pi} a(t) d t,\left\|a(\cdot)-\frac{1}{\pi} \int_{0}^{\pi} a(t) d t\right\|_{L^{1}(0, \pi)}
$$

It is clear that the results given in Theorem 2.3 are of a different nature (see 31 and the translator's note in 25]). In fact, our results are similar to those obtained by Krein [25] by using a different procedure. However, Krein assumed $k=p^{2}$ and an strict inequality for $\|a\|_{L^{1}(0, \pi)}$ in (2.40) (see Theorem 9 in [25]). By using Theorem 2.2 we can assume a non strict inequality in (2.40) since the constant $\tilde{\gamma}_{1, n}$ is not attained.

Finally, if for a given function $a \in L_{\pi}(\mathbb{R}, \mathbb{R})$ we know that $a$ satisfies (2.41), the result given in Theorem 2.3 is more precise than Krein's result since the function

$$
k \pi+k^{1 / 2} 2(p+1) \cot \frac{k^{1 / 2} \pi}{2(p+1)}, k \in\left[p^{2},(p+1)^{2}\right]
$$

is strictly increasing with respect to $k$.

\section{Systems of ORDINARY DIFFERENTIAL EQUATIONS}

In this section we present some results on Lyapunov constants and stability properties for systems of equations

$$
u^{\prime \prime}(t)+P(t) u(t)=0, t \in \mathbb{R},
$$

where the matrix function $P(\cdot)$ is $T$-periodic.

In regard to stability properties of (3.1), the results proved by Krein in 25 show that the problem is closely related to Lyapunov inequalities. In [25], the author assumes that $P(\cdot) \in \Lambda$, where $\Lambda$ is defined as 
The set of real $n \times n$ symmetric matrix valued function $P(\cdot)$, with continuous and $T$-periodic element functions $p_{i j}(t), 1 \leq i, j \leq n$, such that (3.1) has not nontrivial con-

$$
\int_{0}^{T}\langle P(t) k, k\rangle d t \geq 0, \forall k \in \mathbb{R}^{n}
$$

Here, $\langle\cdot, \cdot\rangle$ denotes the usual inner product in $\mathbb{R}^{n}$.

Let us remark that, in the scalar case, the previous hypotheses imply that $\lambda_{0}(P)<0$, where $\lambda_{0}(P)$ is the first eigenvalue for the periodic problem. Therefore, and as in the scalar case, to have some stability property for the system (3.1), it is necessary another condition involving the antiperiodic boundary value problem.

The system (3.1) is said to be stably bounded (25]) if there exists $\varepsilon=$ $\varepsilon(P) \in \mathbb{R}^{+}$, such that all solutions of the system

$$
u^{\prime \prime}(t)+Q(t) u(t)=0, t \in \mathbb{R},
$$

are bounded for all matrix function $Q(\cdot) \in \Lambda$, satisfying

$$
\max _{1 \leq i, j \leq n} \int_{0}^{T}\left|p_{i j}(t)-q_{i j}(t)\right| d t<\varepsilon
$$

that is, for $L_{1}$ small perturbations of the matrix function $P(\cdot)$.

Krein proved that if $P(\cdot) \in \Lambda$, then the system (3.1) is stably bounded if $\lambda_{1}>1$, where $\lambda_{1}$ is the smallest positive eigenvalue of the antiperiodic eigenvalue problem

$$
u^{\prime \prime}(t)+\lambda P(t) u(t)=0, t \in \mathbb{R}, u(0)+u(T)=u^{\prime}(0)+u^{\prime}(T)=0 .
$$

The result given by Krein is a nice theoretical result, but for systems of equations, and assuming $P(\cdot) \in \Lambda$, it is not easy to give sufficient conditions to ensure the property $\lambda_{1}>1$. In this section, we establish some new conditions to get this. Previously, we need to characterize the best $L_{p}$ Lyapunov constants as the minimum of some special minimization problems (constrained or unconstrained, depending on the boundary value problem that we are considering).

A key point is that these minimization problems involve only appropriate functionals defined on some subsets of the corresponding Sobolev space, without any reference to the function $a(\cdot)$ considered in the definition of the Lyapunov constants (for instance, for the case $p=1$, see the definition of the constants $\gamma_{1, n}$ and $\tilde{\gamma}_{1, n}$ in (2.8) and (2.10), respectively).

\section{1. $L_{p}$ Lyapunov constants and minimization problems for ODE.}

This subsection will be concerned with some preliminary results on Lyapunov inequalities for the antiperiodic boundary value problem

$$
u^{\prime \prime}(t)+a(t) u(t)=0, t \in(0, T), u(0)+u(T)=u^{\prime}(0)+u^{\prime}(T)=0,
$$


where $a \in L_{T}(\mathbb{R}, \mathbb{R})$. We consider the antiperiodic problem because we will use these results in the next subsection, but the same idea may be applied to other boundary value problems with Neumann, mixed, Dirichlet, periodic conditions, etc. (see [6], [7] [11, [14], [15])).

As in the case $p=1$, if we define the sets

$$
\Lambda^{\text {ant }}=\left\{a \in L_{T}(\mathbb{R}, \mathbb{R}): \text { (3.4) has nontrivial solutions }\right\},
$$

then for each $p$ with $1 \leq p \leq \infty$, we can define the $L^{p}$ Lyapunov constant $\beta_{p}^{a n t}$ for the antiperiodic problem, as the real number

$$
\beta_{p}^{\text {ant }} \equiv \inf _{a \in \Lambda^{\text {ant }} \cap L^{p}(0, T)}\left\|a^{+}\right\|_{p}
$$

where

$$
\left\|a^{+}\right\|_{p}=\left(\int_{0}^{T}\left|a^{+}(t)\right|^{p} d t\right)^{1 / p}, 1 \leq p<\infty ;\left\|a^{+}\right\|_{\infty}=\text { sup ess } a^{+} .
$$

An explicit expression for the constant $\beta_{p}^{a n t}$, as a function of $p$ and $T$, has been obtained in [39] (see also [6], [10] and [36] for the case of Neumann, mixed and Dirichlet boundary conditions, respectively). As mentioned earlier, in the next theorem we characterize the $L^{p}$ Lyapunov constant $\beta_{p}^{a n t}$, as a minimum of a convenient minimization scalar problem. This will allow the extension of the results to systems of equations. On the other hand, the next theorem is fundamental to intuit the kind of the results we can expect in PDE which will be considered in the next section.

Theorem 3.1. If $1 \leq p \leq \infty$ is a given number, let us define the sets $X_{p}^{\text {ant }}$ and the functional $I_{p}^{\text {ant }}: X_{p}^{\text {ant }} \backslash\{0\} \rightarrow \mathbb{R}$, as

$$
\begin{gathered}
X_{p}^{a n t}=\left\{v \in H^{1}(0, T): v(0)+v(T)=0\right\}, 1 \leq p \leq \infty, \\
I_{1}^{a n t}(v)=\frac{\int_{0}^{T} v^{\prime 2}}{\|v\|_{\infty}^{2}}, \quad I_{\infty}^{\text {ant }}(v)=\frac{\int_{0}^{T} v^{\prime 2}}{\int_{0}^{T} v^{2}} \\
I_{p}^{a n t}(v)=\frac{\int_{0}^{T} v^{\prime 2}}{\left(\int_{0}^{T}|v|^{\frac{2 p}{p-1}}\right)^{\frac{p-1}{p}}}, \text { if } 1<p<\infty .
\end{gathered}
$$

Then, the $L_{p}$ Lyapunov constant $\beta_{p}^{a n t}$ defined in (3.6), satisfies

$$
\beta_{p}^{a n t}=\min _{X_{p}^{a n t} \backslash\{0\}} I_{p}^{a n t}, \quad 1 \leq p \leq \infty .
$$


Proof. In the proof, only the case $1<p<\infty$ is considered (see [14 for the other cases). In summary, we apply the following ideas:

(1) The demonstration that the infimum of the functional $I_{p}^{a n t}$ is attained on $X_{p}^{a n t} \backslash\{0\}$ is standard: we prove the existence of bounded minimizing sequences and then we use the weak lower semicontinuity property of the functional $I_{p}^{a n t}$.

(2) Once we have proved the existence of global minimum, we apply the Lagrange multiplier theorem to obtain the second order (nonlinear) differential equation which must be satisfied by the function $u_{0} \in$ $X_{p}^{a n t} \backslash\{0\}$ where the minimum of $I_{p}^{a n t}$ is attained (Euler equation).

(3) Finally, we integrate the mentioned Euler equation. This is not an easy task and here, again, the number and distribution of the zeros of $u_{0}$ and $u_{0}^{\prime}$ is of an important help.

To carry out the above ideas, let us denote

$$
M_{p}=\inf _{X_{p}^{a n t} \backslash\{0\}} I_{p}^{a n t} .
$$

If $\left\{u_{n}\right\} \subset X_{p}^{a n t} \backslash\{0\}$ is a minimizing sequence, since the sequence $\left\{k_{n} u_{n}\right\}, k_{n} \neq$ 0 , is also a minimizing sequence, we can assume without loos of generality that $\int_{0}^{T}\left|u_{n}\right|^{\frac{2 p}{p-1}}=1$. Then $\left\{\int_{0}^{T}\left|u_{n}^{\prime 2}\right|\right\}$ is also bounded. Moreover, for each $u_{n}$ there is $x_{n} \in[0, T]$ such that $u_{n}\left(x_{n}\right)=0$. Therefore, $\left\{u_{n}\right\}$ is bounded in $H^{1}(0, T)$. So, we can suppose, up to a subsequence, that $u_{n} \rightarrow u_{0}$ in $H^{1}(0, T)$ and $u_{n} \rightarrow u_{0}$ in $C[0, L]$ (the space of continuous functions in $[0, L]$ with the uniform norm). The strong convergence in $C[0, L]$ gives us $u_{0}(0)+u_{0}(T)=0$. Therefore, $u_{0} \in X_{p}^{a n t} \backslash\{0\}$. The weak convergence in $H^{1}(0, T)$ implies $I_{p}^{a n t}\left(u_{0}\right) \leq \liminf I_{p}^{\text {ant }}\left(u_{n}\right)=M_{p}$. Then $u_{0}$ is a minimizer.

We conclude,

$$
H^{\prime}\left(u_{0}\right)(v)=0, \forall v \in H^{1}(0, T) \text { such that } v(0)+v(T)=0 .
$$

Here $H: H^{1}(0, T) \rightarrow \mathbb{R}$ is defined by

$$
H(u)=\int_{0}^{T} u^{\prime 2}-M_{p}\left(\int_{0}^{T}|u|^{\frac{2 p}{p-1}}\right)^{\frac{p-1}{p}}
$$

This implies that $u_{0}$ satisfies the Euler equation

$$
\begin{gathered}
u_{0}^{\prime \prime}(t)+A_{p}\left(u_{0}\right)\left|u_{0}(t)\right|^{\frac{2}{p-1}} u_{0}(t)=0, t \in(0, T), \\
u_{0}(0)+u_{0}(T)=0, u_{0}^{\prime}(0)+u_{0}^{\prime}(T)=0,
\end{gathered}
$$

where

$$
A_{p}\left(u_{0}\right)=M_{p}\left(\int_{0}^{T}\left|u_{0}\right|^{\frac{2 p}{p-1}}\right)^{\frac{-1}{p}}
$$

Since the function $a(t) \equiv A_{p}\left(u_{0}\right)\left|u_{0}(t)\right|^{\frac{2}{p-1}}$ satisfies $a(0)=a(T)$, it is not restrictive to assume that, additionally, $u_{0}(0)=u_{0}(T)=0$. Euler equation 
(3.10) can be integrated, using similar ideas to those considered in [6] Lemma 2.7 and [7] Theorem 2.1, for the Neumann problem. Then, we deduce

$$
M_{p}=\frac{4 n^{2} I^{2} p}{T^{2-\frac{1}{p}}(p-1)^{1-\frac{1}{p}}(2 p-1)^{1 / p}},
$$

where $I=\int_{0}^{1} \frac{d s}{\left(1-s^{\frac{2 p}{p-1}}\right)^{1 / 2}}$ and $n \in \mathbb{N}$ is such that we denote the zeros of $u_{0}$ in $[0, T]$ by $0=x_{0}<x_{2}<\ldots<x_{2 n}=T$ and the zeros of $u_{0}^{\prime}$ in $(0, T)$ by $x_{1}<x_{3}<\ldots<x_{2 n-1}$.

For the antiperiodic problem, (and for the Neumann and Dirichlet problem), $n \geq 1$ and any value $n \geq 1$ is possible. The conclusion is that

$$
M_{p}=\frac{4 I^{2} p}{T^{2-\frac{1}{p}}(p-1)^{1-\frac{1}{p}}(2 p-1)^{1 / p}},
$$

which is the same constant as in the Neumann and Dirichlet problem. Finally, in [39] it is shown that this is, exactly, the $L_{p}$ Lyapunov constant for the antiperiodic problem. The theorem is proved.

3.2. Stability for linear periodic systems. In this subsection we present some $L_{p}$ conditions $(1 \leq p \leq \infty)$ which allow to prove that the smallest positive eigenvalue of the antiperiodic eigenvalue problem (3.3) satisfies $\lambda_{1}>1$. As a consequence, if $P(\cdot) \in \Lambda$, the system (3.1) is stably bounded. These conditions are given in terms of the $L^{p}$ norm of appropriate functions $b_{i i}(t), 1 \leq i \leq n$, related to (3.1) and (3.3) through the inequality $P(t) \leq B(t), \forall t \in[0, T]$, where $B(t)$ is a diagonal matrix with entries given by $b_{i i}(t), 1 \leq i \leq n$. These sufficient conditions are optimal in the sense explained in Remark 6 below. Here, the relation $C \leq D$ between $n \times n$ symmetric matrices means that $D-C$ is positive semi-definite.

From now on we assume that the matrix function $P(\cdot) \in \Lambda$ ( $\Lambda$ was defined at the beginning of this section). In this case, $\lambda_{1}$ has a variational characterization (see [25]) given by

$$
\frac{1}{\lambda_{1}}=\max _{y \in G_{T}} \int_{0}^{T}\langle P(t) y(t), y(t)\rangle d t
$$

where

$$
G_{T}=\left\{y \in\left(H^{1}(0, T)\right)^{n}: y(0)+y(T)=0, \sum_{i=1}^{n} \int_{0}^{T}\left(y_{i}^{\prime}(t)\right)^{2} d t=1\right\} .
$$

Theorem 3.2. Let $P(\cdot) \in \Lambda$ be such that there exist a diagonal matrix $B(t)$ with continuous and $T$-periodic entries $b_{i i}(t)$, and $p_{i} \in[1, \infty], 1 \leq i \leq n$, 
satisfying

$$
\begin{gathered}
P(t) \leq B(t), \forall t \in \mathbb{R}, \\
\left\|b_{i i}^{+}\right\|_{p_{i}}<\beta_{p_{i}}^{a n t}, \text { if } p_{i} \in(1, \infty],\left\|b_{i i}^{+}\right\|_{p_{i}} \leq \beta_{p_{i}}^{a n t} \text {, if } p_{i}=1 .
\end{gathered}
$$

Then, the system (3.1) is stably bounded.

Proof. Let $y \in G_{T}$. Then by using the Theorem 3.1 and more specifically the relation (3.9), we have

$$
\begin{gathered}
\int_{0}^{T}\langle P(t) y(t), y(t)\rangle d t \leq \int_{0}^{T}\langle B(t) y(t), y(t)\rangle d t \leq \\
\sum_{i=1}^{n} \int_{0}^{T} b_{i i}(t)\left(y_{i}(t)\right)^{2}(t) d t \leq \sum_{i=1}^{n}\left\|b_{i i}^{+}(t)\right\|_{p_{i}}\left\|y_{i}^{2}\right\|_{\frac{p_{i}}{p_{i}-1}} \leq \\
\leq \sum_{i=1}^{n} \beta_{p_{i}}^{a n t}\left\|y_{i}^{2}\right\|_{\frac{p_{i}}{p_{i}-1}} \leq \sum_{i=1}^{n} \int_{0}^{T}\left(y_{i}^{\prime}(t)\right)^{2} d t=1, \forall y \in G_{T},
\end{gathered}
$$

where

$$
\begin{aligned}
& \frac{p_{i}}{p_{i}-1}=\infty, \quad \text { if } p_{i}=1 \\
& \frac{p_{i}}{p_{i}-1}=1, \quad \text { if } p_{i}=\infty .
\end{aligned}
$$

(3.17) implies $\lambda_{1} \geq 1$. But if $\lambda_{1}=1$, let us choose $y(\cdot)$ as any nontrivial solution of the problem

$$
y^{\prime \prime}(t)+P(t) y(t)=0, t \in \mathbb{R}, y(0)+y(T)=y^{\prime}(0)+y^{\prime}(T)=0 .
$$

Then some component, say $y_{j}$, is nontrivial. If $p_{j} \in(1, \infty],\left(\beta_{p_{j}}^{a n t}-\left\|b_{j j}^{+}\right\|_{p_{j}}\right)\left\|y_{j}^{2}\right\|_{\frac{p_{j}}{p_{j}-1}}>$ 0 and $\left(\beta_{p_{i}}^{a n t}-\left\|b_{i i}^{+}\right\|_{p_{i}}\right)\left\|y_{i}^{2}\right\|_{\frac{p_{i}}{p_{i}-1}} \geq 0, \forall i \neq j$, so that we have a strict inequality in (3.17). This is a contradiction with (3.18). If $p_{j}=1$, we can use a similar reasoning (see 14]). Consequently $\lambda_{1}>1$ and the theorem is proved.

Remark 6. Previous theorem is optimal in the following sense: for any given positive numbers $\gamma_{i}, 1 \leq i \leq n$, such that at least one of them, say $\gamma_{j}$, satisfies

$$
\gamma_{j}>\beta_{p_{j}}^{\text {ant }} \text {, for some } p_{j} \in[1, \infty],
$$

there exists a diagonal $n \times n$ matrix $P(\cdot) \in \Lambda$ with entries $p_{i i}(t), 1 \leq i \leq n$, satisfying $\left\|p_{i i}^{+}\right\|_{p_{i}}<\gamma_{i}, 1 \leq i \leq n$ and such that the system (3.1) is not stable.

To see this, if $\gamma_{j}$ satisfies (3.19), then there exists some continuous and $T$-periodic function $p(t)$, not identically zero, with $\int_{0}^{T} p(t) d t \geq 0$, and $\left\|p^{+}\right\|_{p_{j}}<\gamma_{j}$, such that the scalar problem

$$
w^{\prime \prime}(t)+p(t) w(t)=0,
$$


is not stable (see Theorem 1 in [38]). If we choose

$$
p_{j j}(t)=p(t), \quad p_{i i}(t)=\delta \in \mathbb{R}^{+}, \text {if } i \neq j,
$$

with $\delta$ sufficiently small, then (3.1) is unstable.

Example 1. In the two dimensional case, we show an example where the hypotheses of the Theorem 3.2 may be checked directly by using the elements $p_{i j}$ of the matrix $P(t)$. The example is based on a similar one shown by the authors in [15, in the study of Lyapunov inequalities for elliptic systems (see also Example 2 below).

Let the matrix $P(t)$ be given by

$$
P(t)=\left(\begin{array}{ll}
p_{11}(t) & p_{12}(t) \\
p_{12}(t) & p_{22}(t)
\end{array}\right)
$$

where

$[\mathbf{H} 1]$

$$
\begin{gathered}
p_{i j} \in C_{T}(\mathbb{R}, \mathbb{R}), 1 \leq i, j \leq 2, \\
p_{11}(t) \geq 0, p_{22}(t) \geq 0, \operatorname{det} P(t) \geq 0, \forall t \in \mathbb{R}, \\
\operatorname{det} P(t) \neq 0, \text { for some } t \in \mathbb{R} .
\end{gathered}
$$

Here $C_{T}(\mathbb{R}, \mathbb{R})$ denotes the set of real, continuous and $T$-periodic functions defined in $\mathbb{R}$ and $\operatorname{det} P(t)$ means the determinant of the matrix $P(t)$.

In addition, let us assume that there exist $p_{1}, p_{2} \in(1, \infty]$ such that

$$
\left\|p_{11}\right\|_{p_{1}}<\beta_{p_{1}}^{\text {ant }}, \quad\left\|p_{22}+\frac{p_{12}^{2}}{\beta_{p_{1}}^{a n t}-\left\|p_{11}\right\|_{p_{1}}}\right\|_{p_{2}}<\beta_{p_{2}}^{\text {ant }} .
$$

Then (3.1) is stably bounded (see [14]) for the details).

Let us observe that from (3.21) we deduce

$$
\left\|p_{11}\right\|_{p_{1}}<\beta_{p_{1}}^{a n t}, \quad\left\|p_{22}\right\|_{p_{2}}<\beta_{p_{2}}^{a n t} .
$$

As a consequence, the uncoupled system

$$
v^{\prime \prime}(t)+R(t) v(t)=0, \quad t \in \mathbb{R},
$$

where

$$
R(t)=\left(\begin{array}{cc}
p_{11}(t) & 0 \\
0 & p_{22}(t)
\end{array}\right)
$$

is stably bounded. Therefore, by using the definition of stably bounded system, (3.1) is stably bounded for any continuous and $T$-periodic function $p_{12}$ with sufficiently small $L_{1}-$ norm. However, (3.21) does not imply, necessarily, that the $L_{1}$-norm of the function $p_{12}$ is necessarily small. 


\section{Scalar Partial Differential Equations}

In this section we consider the linear problem with Neumann boundary conditions

$$
\left.\begin{array}{cl}
\Delta u(x)+a(x) u(x)=0, & x \in \Omega \\
\frac{\partial u}{\partial n}(x)=0, & x \in \partial \Omega
\end{array}\right\}
$$

where $\Omega \subset \mathbb{R}^{N}(N \geq 2)$ is a bounded and regular domain and $\frac{\partial}{\partial n}$ is the outer normal derivative on $\partial \Omega$. Our methods are also applicable to the case of Dirichlet boundary conditions in (4.1) and similar results can be obtained.

However, with respect to ODE there is an important difference: in ODE, once we have solved a concrete problem, for example for Dirichlet boundary conditions, by using appropriate changes of variables we can obtain the best Lyapunov constants for other boundary conditions such as Neumann, periodic, antiperiodic, mixed boundary value problems, etc. (see [39]). To the best of our knowledge, we do not know similar results for PDE and consequently, the problem must be solved differently. We overcome this difficulty characterizing the best constant as the minimum of some convenient minimization problems as it was done for ODE in Theorem 3.1 for the case of antiperiodic boundary conditions.

For Neumann problems like (4.1) the characterization must be established by using some especial constrained minimization problems, a fact that does not occur in the case of Dirichlet boundary conditions (see Lemma 4.2 and Remark 5 in [9] and Theorem 5.1 below).

First we treat the scalar linear problem (4.1). Then we apply the linear study to nonlinear resonant problems by using the Schauder fixed point theorem (see Theorem 4.2). Analogous results can be obtained for nonlinear resonant problems for ODE (see [6], [10], [12] and [13]).

\subsection{Lyapunov constants for scalar partial differential equations.} This subsection will be concerned with the linear boundary value problem (4.1). Remember that $\Omega \subset \mathbb{R}^{N}$ is a bounded and regular domain, and we assume $a \in \Sigma$, where

$\Sigma=\left\{a \in L^{\infty}(\Omega) \backslash\{0\}: \int_{\Omega} a(x) d x \geq 0\right.$ and (4.1) has nontrivial solutions $\}$

As in the ordinary case, the positive eigenvalues of the eigenvalue problem

$$
\left.\begin{array}{cl}
\Delta u(x)+\lambda u(x)=0, & x \in \Omega \\
\frac{\partial u(x)}{\partial n}=0, & x \in \partial \Omega
\end{array}\right\}
$$

belong to $\Sigma$ and therefore, the quantity

$$
\beta_{p} \equiv \inf _{a \in \Sigma}\left\|a^{+}\right\|_{p}, 1 \leq p \leq \infty
$$

is well defined. 
Theorem 4.1. The following statements hold:

(1) $\beta_{1}=0$ and $\beta_{\infty}=\lambda_{1}, \forall N \geq 2$. Here $\lambda_{1}$ denotes the first positive eigenvalue of the eigenvalue problem (4.3).

(2) If $N=2, \beta_{p}>0 \Leftrightarrow p \in(1, \infty]$.

If $N \geq 3, \beta_{p}>0 \Leftrightarrow p \in\left[\frac{N}{2}, \infty\right]$.

If $N \geq 2$ and $\frac{N}{2}<p \leq \infty$, then $\beta_{p}$ is attained.

(3) The mapping $\left(\frac{N}{2}, \infty\right) \rightarrow \mathbb{R}, p \mapsto \beta_{p}$, is continuous and the mapping $\left[\frac{N}{2}, \infty\right] \rightarrow \mathbb{R}, p \mapsto|\Omega|^{-1 / p} \beta_{p}$, is strictly increasing. Here, $|\Omega|$ denotes the Lebesgue measure of the domain $\Omega$.

Proof. We summarize the main ideas:

(1) If $N \geq 3$ and $\frac{N}{2}<p<\infty$, the ideas are the same as in the ordinary case (see Theorem 2.1 in [6]). In fact, since $\frac{N}{2}<p$, then $\frac{2 p}{p-1}<$ $\frac{2 N}{N-2}$ and consequently, the embedding of the Sobolev space $H^{1}(\Omega)$ into $L^{2 p / p-1}(\Omega)$ is compact.

(2) If $N \geq 3$ and $p=\frac{N}{2}$, then $\frac{2 p}{p-1}=\frac{2 N}{N-2}$ and the embedding $H^{1}(\Omega) \subset$ $L^{2 N / N-2}(\Omega)$ is continuous but not compact. This implies that the infimum $\beta_{p}>0$, but we do not know if $\beta_{p}$ is a minimum.

(3) If $N \geq 3$ and $1 \leq p<\frac{N}{2}$, we prove that $\beta_{p}=0$ by finding appropriate minimizing sequences. Roughly speaking, the main idea is to take first a function $u$ and to calculate the corresponding function $a$ for which $u$ is a solution of

$$
\Delta u(x)+a(x) u(x)=0, x \in \Omega, \quad \frac{\partial u(x)}{\partial n}=0, \quad x \in \partial \Omega
$$

Obviously, if $u$ is smooth enough, then we must impose two conditions: i) $\frac{\partial u}{\partial n}=0$ on $\partial \Omega$, ii) The zeros of $u$ are also zeros of $\Delta u$. For instance, if $\Omega=B(0,1)$ we can take radial functions $u(x)=f(|x|)$ of the form $f(r)=\alpha r^{-\delta}-\beta r^{-\gamma},(\delta>0, \gamma>0,0<r<1)$.

The case of general bounded regular domains $\Omega$ can be treated as follows: first of all, note that if we define $\Omega+x_{0}=\left\{x+x_{0} ; x \in \Omega\right\}$ (for arbitrary $x_{0} \in \mathbb{R}^{N}$ ), then $\beta_{p}\left(\Omega+x_{0}\right)=\beta_{p}(\Omega)$. On the other hand, if we define $r \Omega=\{r x ; x \in \Omega\}$ (for arbitrary $r \in \mathbb{R}^{+}$), then $\beta_{p}(r \Omega)=r^{\frac{N}{p}-2} \beta_{p}(\Omega)$. Hence $\beta_{p}(\Omega)=0 \Leftrightarrow \beta_{p}\left(r \Omega+x_{0}\right)=0$. Then, we can suppose without loss of generality that $\bar{B}(0,1) \subset \Omega$.

(4) If $N=2$ and $p=1$, the ideas are the same as in the previous step, but we use the fundamental solution $\ln |x|$ to find appropriate minimizing sequences (see Lemma 3.2 in [9]).

(5) If $N=2$, the embedding $H^{1}(\Omega) \subset L^{q}(\Omega)$ is compact $\forall q \in[1, \infty)$ and therefore, if $1<p<\infty$, the ideas are the same as in the ordinary case. 
(6) The case $p=\infty$ is trivial if we use the variational characterization of $\lambda_{1}$.

Remark 7 . The set $\Sigma$ defined in (4.2) can be replaced by

If $N \geq 3$,

$\Sigma=\left\{a \in L^{\frac{N}{2}}(\Omega) \backslash\{0\}: \int_{\Omega} a(x) d x \geq 0\right.$ and (4.1) has nontrivial solutions $\}$

and if $N=2$,

$\Sigma=\left\{a: \Omega \rightarrow \mathbb{R}\right.$ s. t. $\exists q \in(1, \infty]$ with $a \in L^{q}(\Omega) \backslash\{0\}, \int_{\Omega} a(x) d x \geq 0$ and (4.1)

has nontrivial solutions

Remark 8. In the definition of the set $\Sigma$ we have imposed $\int_{\Omega} a \geq 0$. This is not a technical but a natural assumption for Neumann boundary conditions (an also for periodic boundary conditions in the ordinary case. See Section 2 in [14]). Otherwise, the corresponding infimum will be always zero. To see this, note that if $u \in H^{1}(\Omega)$ is a positive nonconstant solution of (4.1) and we consider $v=\frac{1}{u}$ as test function in the weak formulation, we obtain

which implies

$$
\int_{\Omega} \nabla u \cdot \nabla\left(\frac{1}{u}\right)=\int_{\Omega} a u \frac{1}{u}
$$

$$
\int_{\Omega} a=-\int_{\Omega} \frac{|\nabla u|^{2}}{u^{2}}<0 .
$$

With this in mind, if we take a nonconstant $u_{0} \in C^{2}(\bar{\Omega})$ such that $\frac{\partial u_{0}}{\partial n}(x)=0, \forall x \in \partial \Omega$ then, for large $n \in \mathbb{N}$, we have that $u_{n}=u_{0}+n$ is a positive nonconstant solution of (4.1), with $a_{n}=\frac{-\Delta u_{0}}{u_{0}+n}$. Clearly $\int_{\Omega} a_{n}<0$ and $\left\|a_{n}\right\|_{p} \rightarrow 0$ as $n \rightarrow \infty$ for every $1 \leq p \leq \infty$.

Remark 9. A property similar to the last part of the previous theorem is valid for ODE (see, for instance, Theorem 2.1 in [6] for the case of Neumann boundary conditions).

4.2. Resonant nonlinear scalar problems for PDE. The results that we have obtained for linear problem (4.1) can be useful for the study of nonlinear boundary value problems of the form

$$
\left.\begin{array}{cl}
\Delta u(x)+f(x, u(x))=0, & x \in \Omega \\
\frac{\partial u}{\partial n}(x)=0, & x \in \partial \Omega
\end{array}\right\}
$$

In fact, this is common in analysis: to study nonlinear problems with the help of convenient linearized problems (implicit function theorem, inverse function theorem, bifurcation theory, etc.) 
As in the previous subsection we assume that $\Omega \subset \mathbb{R}^{N}(N \geq 2)$ is a bounded and regular domain and the function $f: \bar{\Omega} \times \mathbb{R} \rightarrow \mathbb{R},(x, u) \mapsto f(x, u)$, satisfies the condition

(H) $f, f_{u}$ are Caratheodory functions i.e. $f(x, u), f_{u}(x, u)$ are measurable in $x$ for every $u$ and continuous in $u$ for almost every $x$ and $0 \leq f_{u}(x, u)$ in $\bar{\Omega} \times \mathbb{R}$.

The existence of a solution of (4.5) implies

$$
\int_{\Omega} f\left(x, s_{0}\right) d x=0
$$

for some $s_{0} \in \mathbb{R}$. Trivially, conditions $(\mathrm{H})$ and (4.6) are not sufficient for the existence of solutions of (4.5). Indeed, consider the problem

$$
\left.\begin{array}{cl}
-\Delta u(x)=\lambda_{1} u(x)+\varphi_{1}(x), & x \in \Omega \\
\frac{\partial u}{\partial n}(x)=0, & x \in \partial \Omega
\end{array}\right\}
$$

where $\varphi_{1}$ is a nontrivial eigenfunction associated to $\lambda_{1}$ (remember that $\lambda_{1}$ is the first positive eigenvalue of the eigenvalue problem (4.3)). The function $f(x, u)=\lambda_{1} u+\varphi_{1}(x)$ satisfies $(\mathbf{H})$ and (4.6), but the Fredholm alternative theorem shows that there is no solution of (4.7).

If, in addition to $(\mathbf{H})$ and (4.6),$f$ satisfies a non-uniform non-resonance condition of the type

(h1) $f_{u}(x, u) \leq \beta(x)$ in $\bar{\Omega} \times \mathbb{R}$ with $\beta(x) \leq \lambda_{1}$ in $\Omega$ and $\beta(x)<\lambda_{1}$ in a subset of $\Omega$ of positive measure,

then it has been proved in [34 that (4.5) has solution. Let us observe that supplementary condition (h1) is given in terms of $\|\beta\|_{\infty}$. In the next result, we provide new supplementary conditions in terms of $\|\beta\|_{p}$, where $N / 2<p \leq \infty$, obtaining a generalization of Theorem 2 in 34. For the case $N / 2<p<\infty$ it is clear that the function $f_{u}(x, u)$ may cross an arbitrary number of eigenvalues of the corresponding linear problem, as long as certain $L_{p}$-norms are controlled.

In the proof, the basic idea is to combine the results obtained in the previous section with the Schauder's fixed point theorem. However we omit this proof here since a more general one will be given in the case of systems of equations (see Theorem 5.3).

Theorem 4.2. Let $\Omega \subset \mathbb{R}^{N}(N \geq 2)$ be a bounded and regular domain and $f: \bar{\Omega} \times \mathbb{R} \rightarrow \mathbb{R},(x, u) \mapsto f(x, u)$, satisfying:

(1) $f, f_{u}$ are Caratheodory functions and $f(\cdot, 0) \in L^{\infty}(\Omega)$.

(2) There exists a function $\beta \in L^{\infty}(\Omega)$, satisfying

$$
0 \leq f_{u}(x, u) \leq \beta(x) \text { in } \bar{\Omega} \times \mathbb{R}
$$

and such that for some $p, N / 2<p \leq \infty$, we have $\|\beta\|_{p}<\beta_{p}$ where $\beta_{p}$ is given by Theorem 4.1. 


$$
\exists s_{0} \in \mathbb{R} \text { s.t. } \int_{\Omega} f\left(x, s_{0}\right) d x=0, \text { and } f_{u}(x, u(x)) \not \equiv 0, \forall u \in C(\bar{\Omega})
$$

Then problem (4.5) has a unique solution.

\section{Systems of Partial Differential equations.}

In this section we deal with elliptic systems of the form

$$
\Delta u(x)+A(x) u(x)=0, x \in \Omega, \quad \frac{\partial u(x)}{\partial n}=0, x \in \partial \Omega,
$$

As previously, $\Omega \subset \mathbb{R}^{N}, N \geq 2$ is a bounded and regular domain and $\frac{\partial}{\partial n}$ is the outer normal derivative on $\partial \Omega$. On the other hand, $A \in \Sigma_{*}$, where $\Sigma_{*}$ is defined as

The set of real $n \times n$ symmetric matrix valued function $A(\cdot)$, with continuous element functions $a_{i j}(x), 1 \leq i, j \leq n, x \in$ $\bar{\Omega}$, such that (5.1) has not nontrivial constant solutions and

$$
\int_{\Omega}<A(x) k, k>d x \geq 0, \forall k \in \mathbb{R}^{n} .
$$

In (5.1), $u \in\left(H^{1}(\Omega)\right)^{n}$, the usual Sobolev space.

Similar to the ordinary case, to be able to extend to the case of systems of equations the obtained results in the scalar case, a key point is the characterization of the best $L_{p}$ Lyapunov constant for the scalar given problem, as a minimum of some especial minimization problems defined in appropriate subsets $X_{p}, N / 2<p \leq \infty$, of the Sobolev space $H^{1}(\Omega)$. This is done in the next subsection. Since 0 is the first eigenvalue for Neumann problem, it is necessary to impose an additional restriction to the definition of the spaces $X_{p}, N / 2<p \leq \infty$ (see Remark 8 above). This is not necessary in the case of Dirichlet one.

5.1. Lyapunov constants for PDE and constrained minimization problems. In this subsection we consider $N \geq 2$. Remember that if $\frac{N}{2}<$ $p \leq \infty$ and

$\Sigma=\left\{a \in L^{\infty}(\Omega) \backslash\{0\}: \int_{\Omega} a(x) d x \geq 0\right.$ and (5.1) has nontrivial solutions $\}$

then

$$
\beta_{p} \equiv \min _{a \in \Sigma}\left\|a^{+}\right\|_{p}, \quad N / 2<p \leq \infty .
$$


The next theorem can be proved by using the same ideas as in the ordinary case (see Lemma 2.5 and Lemma 2.6 in [6] and Lemma 4.1, Lemma 4.2 and Lemma 4.3 in [9]).

Theorem 5.1. If $\frac{N}{2}<p \leq \infty$ is a given number, let us define the set $X_{p}$ and the functional $I_{p}$ as

$$
\begin{gathered}
X_{p}=\left\{v \in H^{1}(\Omega): \int_{\Omega}|v|^{\frac{2}{p-1}} v=0\right\}, \text { if } \frac{N}{2}<p<\infty, \\
I_{p}: X_{p} \backslash\{0\} \rightarrow \mathbb{R}, I_{p}(v)=\frac{\int_{\Omega}|\nabla v|^{2}}{\left(\int_{\Omega}|v|^{\frac{2 p}{p-1}}\right)^{\frac{p-1}{p}}}, \text { if } \frac{N}{2}<p<\infty, \\
X_{\infty}=\left\{v \in H^{1}(\Omega): \int_{\Omega} v=0\right\}, \\
I_{\infty}: X_{\infty} \backslash\{0\} \rightarrow \mathbb{R}, I_{\infty}(v)=\frac{\int_{\Omega}|\nabla v|^{2}}{\int_{\Omega} v^{2}}
\end{gathered}
$$

Then

$$
\beta_{p} \equiv \min _{X_{p} \backslash\{0\}} I_{p}, \frac{N}{2}<p \leq \infty,
$$

Remark 10. Let us observe that, as in the ordinary case, $\beta_{\infty}=\lambda_{1}$, the first strictly positive eigenvalue of the Neumann eigenvalue problem in the domain $\Omega$. Consequently, for PDE it seems difficult to obtain explicit expressions for $\beta_{p}$, as a function of $p, \Omega$ and $N$, at least for general domains. However, for ODE, it is possible: see (3.13).

In the next theorem we provide for each $p$, with $p \in(N / 2, \infty]$, optimal necessary conditions for boundary value problem (5.1) to have only the trivial solution. These conditions are given in terms of the $L^{p}$ norm of appropriate functions $b_{i i}(x), 1 \leq i \leq n$, related to $A(x)$ through the inequality $A(x) \leq B(x), \forall x \in \bar{\Omega}$, where $B(x)$ is a diagonal matrix with entries given by $b_{i i}(x), 1 \leq i \leq n$. In particular, we can use different $L_{p_{i}}$ criteria for each $1 \leq i \leq n$ and this confers a great generality on our results. Since we provide $L^{p}$ restrictions with $p \in(N / 2, \infty)$, the eigenvalues of the matrix $A(x)$ may cross an arbitrary number of eigenvalues of the problem

$$
\Delta u(x)+\lambda u(x)=0, x \in \Omega, \frac{\partial u(x)}{\partial n}=0, x \in \partial \Omega,
$$

as long as certain $L^{p}$-norms of the functions $b_{i i}$ are controlled. The detailed proof may be seen in [15]. 
Theorem 5.2. Let $A(\cdot) \in \Sigma_{*}$ be such that there exist a diagonal matrix $B(x)$ with continuous entries $b_{i i}(x)$, and numbers $p_{i} \in(N / 2, \infty], 1 \leq i \leq n$, which fulfil

$$
\begin{gathered}
A(x) \leq B(x), \forall x \in \bar{\Omega} \\
\left\|b_{i i}^{+}\right\|_{p_{i}}<\beta_{p_{i}}, \quad 1 \leq i \leq n .
\end{gathered}
$$

Then, there exists no nontrivial solution of the vector boundary value problem (5.1).

Remark 11. The previous theorem is optimal in the following sense: for any given positive numbers $\gamma_{i}, 1 \leq i \leq n$, such that at least one of them, say $\gamma_{j}$, satisfies

$$
\gamma_{j}>\beta_{p_{j}}, \text { for some } p_{j} \in(N / 2, \infty],
$$

there exists a diagonal $n \times n$ matrix $A(\cdot) \in \Sigma_{*}$ with continuous entries $a_{i i}(x), 1 \leq i \leq n$, satisfying $\left\|a_{i i}^{+}\right\|_{p_{i}}<\gamma_{i}, 1 \leq i \leq n$ and such that the boundary value problem (5.1) has nontrivial solutions.

In the next example we consider the case of a system with two equations. This example is similar to that considered in Remark 1 when stability properties for a two dimensional systems were obtained. Our aim here is to prove that the unique solution of (5.1) is the trivial one, showing the multiple applications of Lyapunov inequalities.

Example 2. Let the matrix $A(x)$ be given by

$$
A(x)=\left(\begin{array}{ll}
a_{11}(x) & a_{12}(x) \\
a_{12}(x) & a_{22}(x)
\end{array}\right)
$$

where

$$
a_{i j} \in C(\bar{\Omega}), 1 \leq i, j \leq 2,
$$

$$
a_{11}(x) \geq 0, a_{22}(x) \geq 0, a_{11}(x) a_{22}(x) \geq a_{12}^{2}(x), \forall x \in \bar{\Omega},
$$

$\operatorname{det} A(x) \neq 0$, for some $x \in \bar{\Omega}$.

In addition, let us assume that there exist $p_{1}, p_{2} \in(N / 2, \infty]$ such that

$$
\left\|a_{11}\right\|_{p_{1}}<\beta_{p_{1}}, \quad\left\|a_{22}+\frac{a_{12}^{2}}{\beta_{p_{1}}-\left\|a_{11}\right\|_{p_{1}}}\right\|_{p_{2}}<\beta_{p_{2}} .
$$

Then the unique solution of (5.1) is the trivial one.

To prove the previous affirmation, it is trivial to see that $[\mathbf{H 1}]$ implies that the eigenvalues of the matrix $A(x)$ are both nonnegative, which implies that $A(x)$ is positive semi-definite. Also, since $\operatorname{det} A(x) \neq 0$, for some $x \in \bar{\Omega}$, 
(5.1) has not nontrivial constant solutions. Therefore, $A(\cdot) \in \Sigma_{*}$. Moreover, it is easy to check that for a given diagonal matrix $B(x)$, with continuous entries $b_{i i}(x), 1 \leq i \leq 2$, the relation

$$
A(x) \leq B(x), \forall x \in \bar{\Omega}
$$

is satisfied if and only if $\forall x \in \bar{\Omega}$, we have

$$
\begin{gathered}
b_{11}(x) \geq a_{11}(x), \quad b_{22}(x) \geq a_{22}(x), \\
\left(b_{11}(x)-a_{11}(x)\right)\left(b_{22}(x)-a_{22}(x)\right) \geq a_{12}^{2}(x) .
\end{gathered}
$$

In our case, if we choose

$$
b_{11}(x)=a_{11}(x)+\gamma, b_{22}(x)=a_{22}(x)+\frac{a_{12}^{2}(x)}{\gamma}
$$

where $\gamma$ is any constant such that

$$
\begin{gathered}
0<\gamma<\beta_{p_{1}}-\left\|a_{11}\right\|_{p_{1}}, \\
\left(\frac{1}{\gamma}-\frac{1}{\beta_{p_{1}}-\left\|a_{11}\right\|_{p_{1}}}\right)\left\|a_{12}^{2}\right\|_{p_{2}}<\beta_{p_{2}}-\left\|a_{22}+\frac{a_{12}^{2}}{\beta_{p_{1}}-\left\|a_{11}\right\|_{p_{1}}}\right\|_{p_{2}}
\end{gathered}
$$

then all conditions of Theorem 5.2 are fulfilled and consequently (5.1) has only the trivial solution.

Remark 12. The result given in the previous remark may be seen as a perturbation result in the following sense: let us assume that we have an uncoupled system of the type

$$
\begin{aligned}
& \Delta u_{1}(x)+a_{11}(x) u_{1}(x)=0, x \in \Omega ; \quad \frac{\partial u_{1}(x)}{\partial n}=0 \quad x \in \partial \Omega, \\
& \Delta u_{2}(x)+a_{22}(x) u_{2}(x)=0, x \in \Omega ; \quad \frac{\partial u_{2}(x)}{\partial n}=0 \quad x \in \partial \Omega,
\end{aligned}
$$

where

$$
\begin{gathered}
a_{i i} \in C(\bar{\Omega}), 1 \leq i \leq 2, a_{11}(x) \geq \delta>0, a_{22}(x) \geq \delta, \forall x \in \bar{\Omega} . \\
\quad \exists p_{1}, p_{2} \in(N / 2, \infty]:\left\|a_{11}\right\|_{p_{1}}<\beta_{p_{1}}, \quad\left\|a_{22}\right\|_{p_{2}}<\beta_{p_{2}} .
\end{gathered}
$$

Then it is clear from the scalar results (see Remark 10) that the unique solution of (5.14) is the trivial one (see Corollary 6.1 in [9]). As it is shown in the previous remark, we can ensure the permanence of the uniqueness property (with respect to the existence of solutions) of the coupled system (5.1), for any function $a_{12} \in C(\bar{\Omega})$ with $L^{\infty}$-norm sufficiently small. Here we have considered that the functions $a_{i i}(x), 1 \leq i \leq 2$, are fixed and that the uncoupled system is perturbed by the function $a_{12}(x)$. But it is clear that we may consider, for example, $a_{11}(x), a_{12}(x)$ fixed and $a_{22}(x)$ as the perturbation. 
5.2. Resonant nonlinear systems of PDE. Next we give some new results on the existence and uniqueness of solutions of nonlinear resonant problems. Similar results can be proved for ordinary differential systems; in this last case it is possible to choose the constants $p_{i} \in[1, \infty], 1 \leq i \leq n$. In particular, next theorem is a generalization, for systems of equations, of the main result given in [34 for the Neumann problem. Moreover, it is a generalization (at the two first eigenvalues) of some results given in [1 and [24] where the authors take all the constants $p_{i}=\infty, 1 \leq i \leq n$.

In the proof, the basic idea is to combine the results obtained in the linear case with Schauder's fixed point theorem.

Theorem 5.3. Let $\Omega \subset \mathbb{R}^{N}(N \geq 2)$ be a bounded and regular domain and $G: \bar{\Omega} \times \mathbb{R}^{n} \rightarrow \mathbb{R},(x, u) \rightarrow G(x, u)$ satisfying:

(1) (a) $u \rightarrow G(x, u)$ is of class $C^{2}\left(\mathbb{R}^{n}, \mathbb{R}\right)$ for every $x \in \bar{\Omega}$. (b) $x \rightarrow G(x, u)$ is continuous on $\bar{\Omega}$ for every $u \in \mathbb{R}^{n}$.

(2) There exist continuous matrix functions $A(\cdot), B(\cdot)$, with $B(x)$ diagonal and with entries $b_{i i}(x)$, and $p_{i} \in(N / 2, \infty], 1 \leq i \leq n$, such that

$$
\left.\begin{array}{c}
A(x) \leq G_{u u}(x, u) \leq B(x) \text { in } \bar{\Omega} \times \mathbb{R}^{n}, \\
\left\|b_{i i}^{+}\right\|_{p_{i}}<\beta_{p_{i}}, 1 \leq i \leq n, \\
\int_{\Omega}<A(x) k, k>d x>0, \forall k \in \mathbb{R}^{n} \backslash\{0\}
\end{array}\right\}
$$

Then system

$$
\left.\begin{array}{c}
\Delta u(x)+G_{u}(x, u(x))=0, x \in \Omega, \\
\frac{\partial u(x)}{\partial n}=0, \quad x \in \partial \Omega,
\end{array}\right\}
$$

has a unique solution.

Proof. We first prove uniqueness. Let $v$ and $w$ be two solutions of (5.17). Then, the function $u=v-w$ is a solution of the problem

$$
\Delta u(x)+C(x) u(x)=0, x \in \Omega, \frac{\partial u}{\partial n}=0, x \in \partial \Omega
$$

where $C(x)=\int_{0}^{1} G_{u u}(x, w(x)+\theta u(x)) d \theta$ (see [26], p. 103, for the mean value theorem for the vectorial function $\left.G_{u}(x, u)\right)$. Hence $A(x) \leq C(x) \leq$ $B(x)$ and we deduce that $C(x)$ satisfies all the hypotheses of Theorem 5.2 . Consequently, $u \equiv 0$.

Next we prove existence. First, we write (5.17) in the equivalent form

$$
\left.\begin{array}{cc}
\Delta u(x)+D(x, u(x)) u(x)+G_{u}(x, 0)=0, & \text { in } \Omega, \\
\frac{\partial u}{\partial n}=0, & \text { on } \partial \Omega
\end{array}\right\}
$$


where the function $D: \bar{\Omega} \times \mathbb{R}^{n} \rightarrow \mathcal{M}(\mathbb{R})$ is defined by $D(x, z)=\int_{0}^{1} G_{u u}(x, \theta z) d \theta$. Here $\mathcal{M}(\mathbb{R})$ denotes the set of real $n \times n$ matrices. Let $X=(C(\bar{\Omega}))^{n}$ be with the uniform norm, i.e., if $y(\cdot)=\left(y^{1}(\cdot), \cdots, y^{n}(\cdot)\right) \in X$, then $\|y\|_{X}=$ $\sum_{k=1}^{n}\left\|y^{k}(\cdot)\right\|_{\infty}$. Since

$$
A(x) \leq D(x, z) \leq B(x), \forall(x, z) \in \bar{\Omega} \times \mathbb{R}^{n},
$$

we can apply Theorem 5.2 in order to have a well defined operator $T: X \rightarrow$ $X$, by $T y=u_{y}$, being $u_{y}$ the unique solution of the linear problem

$$
\left.\begin{array}{cc}
\Delta u(x)+D(x, y(x)) u(x)+G_{u}(x, 0)=0, & \text { in } \Omega, \\
\frac{\partial u}{\partial n}=0, & \text { on } \partial \Omega .
\end{array}\right\}
$$

Now, we can show that $T$ is completely continuous and that $T(X)$ is bounded (see Theorem 3.4 in [15] for the details). The Schauder's fixed point theorem provides a fixed point for $T$ which is a solution of (5.17).

\section{Final Remarks}

In this section we briefly discuss some questions related to the previous results. It may be useful to complete the contents of the paper or for future research.

\section{Lyapunov inequalities and disfocality.}

When the Neumann (or periodic) boundary value problem is considered, it is necessary to assume the natural restrictions

$$
a \in L^{1}(0, T) \backslash\{0\}, \quad \int_{0}^{T} a(t) d t \geq 0 .
$$

Otherwise, we obtain trivial Lyapunov inequalities (see Remark 4 in [6] , Remark 4 in [9] and Remark 8 in this paper).

Under the restrictions (6.1), the relation between, for example, Neumann boundary conditions, and disfocality arises in a natural way: if $u \in H^{1}(0, T)$ is any nontrivial solution of

$$
u^{\prime \prime}(t)+a(t) u(t)=0, t \in(0, T), u^{\prime}(0)=u^{\prime}(T)=0
$$

then $u$ must have a zero $c$ in the interval $(0, T)$. In consequence both problems

$$
v^{\prime \prime}(t)+a(t) v(t)=0, t \in(0, c), v^{\prime}(0)=v(c)=0, \quad \mathbf{P M}(\mathbf{0}, \mathbf{c})
$$

and

$$
v^{\prime \prime}(t)+a(t) v(t)=0, t \in(c, L), v(c)=v^{\prime}(L)=0, \quad \mathbf{P M}(\mathbf{c}, \mathbf{L})
$$


have nontrivial solutions.

This simple observation can be used to deduce the following conclusion: if $a \in L^{1}(0, T) \backslash\{0\}$ with $\int_{0}^{T} a \geq 0$ is any function such that for any $c \in(0, T)$, either problem $\mathbf{P M}(\mathbf{0}, \mathbf{c})$ or problem $\mathbf{P M}(\mathbf{c}, \mathbf{L})$ has only the trivial solution, then problem (6.2) has only the trivial solution. This idea is exploited in [10] to obtain new results on the existence and uniqueness of solutions for resonant problems with Neumann boundary conditions (but it is clear from the proof that the same idea can be used for other boundary conditions). For example, by using the $L^{\infty}$ norm of the function $a^{+}$, it can be proved that if

$$
\begin{gathered}
a \in L^{\infty}(0, T) \backslash\{0\}, \int_{0}^{T} a \geq 0 \text { and } \exists t_{0} \in(0, T): \\
\max \left\{t_{0}^{2}\left\|a^{+}\right\|_{L^{\infty}\left(0, t_{0}\right)},\left(T-t_{0}\right)^{2}\left\|a^{+}\right\|_{L^{\infty}\left(t_{0}, T\right)}\right\} \leq \pi^{2} / 4
\end{gathered}
$$

and, in addition, either $a^{+}$is not the constant $\pi^{2} / 4 t_{0}^{2}$ in the interval $\left[0, t_{0}\right]$ or $a^{+}$is not the constant $\pi^{2} / 4\left(T-t_{0}\right)^{2}$ in the interval $\left[t_{0}, T\right]$, then we obtain that (6.2) has only the trivial solution (these kinds of functions $a$ are usually named two step potentials).

Hypothesis $(\mathbf{H})$ is optimal in the sense that if $a^{+}$is the constant $\pi^{2} / 4 t_{0}^{2}$ in the interval $\left[0, t_{0}\right]$ and $a^{+}$is the constant $\pi^{2} / 4\left(T-t_{0}\right)^{2}$ in the interval $\left[t_{0}, T\right]$, then (6.2) has nontrivial solutions.

If $t_{0}=T / 2$, we have the classical result related to the so called nonuniform non-resonance conditions with respect to the first two eigenvalues of the Neumann boundary value problem: if

$$
0 \prec a \prec \frac{\pi^{2}}{T^{2}},
$$

then (6.2) has only the trivial solution $([32,, 33,, 34])$. But if for instance $t_{0} \in(0, T / 2)$ then function $a$ can satisfy $\left\|a^{+}\right\|_{L^{\infty}\left(0, t_{0}\right)}=\pi^{2} /\left(4 t_{0}^{2}\right)$ (which is a quantity greater than $\left.\pi^{2} / T^{2}\right)$ as long as $\left\|a^{+}\right\|_{L^{\infty}\left(t_{0}, T\right)}<\pi^{2} / 4\left(T-x_{0}\right)^{2}$.

These ideas are valid not only for $p=\infty$, but also for $L_{p}$ Lyapunov inequalities and disfocality when $1 \leq p<\infty$ ([10]) and also at higher eigenvalues (12]).

2. $L_{p}$-Lyapunov inequalities for scalar equations at higher eigenvalues, $1<p<\infty$.

In Section 2, we have obtained the best $L_{\infty}-L_{1}$ Lyapunov constant for the periodic and antiperiodic boundary value problems at higher eigenvalues. For example, for the periodic problem we considered the set $\Lambda_{n}$ defined in (2.7) as

(6.3) $\Lambda_{n}=\left\{a \in L_{T}(\mathbb{R}, \mathbb{R}): \lambda_{2 n-1} \prec a\right.$ and (2.1) has nontrivial solutions $\}$ 
and the best $L_{\infty}-L_{1}$ Lyapunov constant was defined as

$$
\gamma_{1, n}=\inf _{a \in \Lambda_{n}}\|a\|_{L^{1}(0, T)}
$$

In a similar way, we can obtained very easily the best $L_{\infty}-L_{\infty}$ Lyapunov constant for the periodic problem, at higher eigenvalues, i.e.,

$$
\gamma_{\infty, n}=\inf _{a \in \Lambda_{n}}\|a\|_{L^{\infty}(0, T)}=\lambda_{2 n+1}
$$

Remember that $\lambda_{2 n-1}=\lambda_{2 n}=\frac{4 n^{2} \pi^{2}}{T^{2}}$ and that $\lambda_{2 n+1}=\lambda_{2 n+2}=\frac{4(n+1)^{2} \pi^{2}}{T^{2}}$.

After this, it is quite natural to consider the same question when $1<p<$ $\infty$, that is, try to calculate explicitly

$$
\gamma_{p, n}=\inf _{a \in \Lambda_{n}}\|a\|_{L^{p}(0, T)}
$$

In this regard, we have developed some ideas but we have no a definitive answer yet. The main difficulty is that the associated minimization problem, defined in (2.14) for the case $p=1$, is now a convex-concave problem which seems difficult to study. However, it would be important to have a positive answer to this question, since in this case, considering $\lim _{p \rightarrow 1^{+}} \gamma_{p, n}$ and $\lim _{p \rightarrow \infty} \gamma_{p, n}$ there would be a natural relationship between the cases $p=1$ and $p=\infty$.

\section{Lyapunov inequalities for systems of equations at higher eigenvalues.}

In Section 3 we have considered systems of equations and we have presented some optimal Lyapunov inequalities but always at the first eigenvalue. For example, for the antiperiodic problem, $\beta_{\infty}^{a n t}$, defined in (3.6) (see also (3.9) ) is equal to $\frac{\pi^{2}}{T^{2}}$, the first eigenvalue of the antiperiodic boundary value problem (1.4) for $a \equiv 0$.

It would be interesting to have $L_{1}$ Lyapunov inequalities for systems of equations at higher eigenvalues. The main difficulty is to obtain a similar characterization, to that given in (3.9) for the antiperiodic problem, for the constant $\tilde{\gamma}_{1, n}$ defined in (2.28).

Finally, but this depends on having been successful or not in the previous remark, we can try to obtain $L_{p}$ Lyapunov inequalities for systems of equations at higher eigenvalues if $1<p<\infty$.

\section{Explicit value of the Lyapunov constant for PDE and sym- metric domains.}

In the PDE case, we defined the Lyapunov constant for the Neumann problem $\beta_{p, \Omega}$ in (4.4) (here we emphasize the dependence on the domain $\Omega$ ).

In particular $\beta_{\infty, \Omega}=\lambda_{1}(\Omega)$, the first positive eigenvalue of the eigenvalue problem (4.3). It is known that except in very special cases, it is not possible 
to calculate explicitly $\lambda_{1}(\Omega)$, so that the same will occurs with $\beta_{p, \Omega}$. However, in Theorem 5.1, $\beta_{p, \Omega}$ is characterized as the minimum value of some minimization problems. It is possible that, for certain types of domains, $\beta_{p, \Omega}$ can be calculated explicitly. This requires a study of the possible symmetries of those functions where the minimum is attained. Anyway, this seems a difficult problem.

Finally the authors are considering in [16] Lyapunov inequalities for PDE at radial higher eigenvalues.

\section{REFERENCES}

[1] P.W. Bates. Solutions of nonlinear elliptic systems with meshed spectra. Nonlinear Anal., 4 (1979), 1023-1030.

[2] G. Borg. Über die Stabilität gewisser Klassen von linearen Differentialgleichungen. Ark. Mat. Astr. Fys., 31 A, 1, (1944), 1-31.

[3] G. Borg. On a Liapounoff criterion of stability. Amer. J. Math., 71 (1949), 67-70.

[4] H. Brezis. Symmetry in nonlinear PDE's. Proc. Sympos. Pure Math. Amer. Math. Soc., Providence, R.I. 65, (1999), 1-12.

[5] H. Brezis and L. Nirenberg. Positive solutions of nonlinear elliptic equations involving critical Sobolev exponents. Comm. Pure Appl. Math. 36, (1983), 437-477.

[6] A. Cañada, J.A. Montero and S. Villegas. Liapunov-type inequalities and Neumann boundary value problems at resonance. Math. Ineq. Appl., 8 (2005),459-475.

[7] A. Cañada, J.A. Montero and S. Villegas. Lyapunov-type inequalities for differential equations. Mediterr. J. Math. 3, (2006), 177-187.

[8] A. Cañada. J. A. Montero and S. Villegas. Lyapunov-type inequalities and applications to PDE. Progr. Nonlinear Differential Equations Appl., 63, 103-110, Birkhäuser, 2005.

[9] A. Cañada, J.A. Montero and S. Villegas. Lyapunov inequalities for partial differential equations. J. Funct. Anal., 237, (2006), 176-193.

[10] A. Cañada and S. Villegas. Optimal Lyapunov inequalities for disfocality and Neumann boundary conditions using $L^{p}$ norms. Discrete and Continuous Dynamical Systems, series A, 20, (2008), 877-888.

[11] A. Cañada and S. Villegas. Optimal Lyapunov inequalities for boundary value problems. J. Math. Inequal. 3, (2009), 631-643.

[12] A. Cañada and S. Villegas. Lyapunov inequalities for Neumann boundary conditions at higher eigenvalues. J. Eur. Math. Soc., 12, (2010), 163-178.

[13] A. Cañada and S. Villegas. Lyapunov inequalities for the periodic boundary value problem at higher eigenvalues. J. Math. Anal. Appl., 376, (2011), 429-442.

[14] A. Cañada and S. Villegas. Stability, resonance and Lyapunov inequalities for periodic conservative systems. Non. Anal., 74 (2011), 1913-1925.

[15] A. Cañada and S. Villegas. Matrix Lyapunov inequalities for ordinary and elliptic partial differential equations. Submitted for publication. arXiv:0906.1093

[16] A. Cañada and S. Villegas Lyapunov inequalities for Partial Differential Equations in the radial case. Submmitted for publication. arXiv:1109.5020

[17] E.A. Coddington and N. Levinson. Theory of Ordinary Differential Equations. McGraw-Hill, 1955.

[18] G. Croce and B. Dacorogna. On a generalized Wirtinger inequality. Discrete and Continuous Dynamical Systems, 9, (2003), 1329-1341.

[19] B. Dacarogna,W. Gangbo and N. Subía. Sur une généralisation de l'inégalité de Wirtinger. Ann. Inst. Henri Poincaré, Anal. Non Linéaire, 9, (1992), 29-50.

[20] G. López and J.A. Montero. Neumann boundary value problems across resonance. ESAIM Control Optim. Calc. Var. 12, (2006), 398-408. 
[21] J.K. Hale. Ordinary Differential Equations. John Wiley and Sons, Inc., 1969.

[22] P. Hartman. Ordinary Differential Equations. John Wiley and Sons Inc., 1964.

[23] W. Huaizhong and L. Yong. Existence and uniqueness of periodic solutions for Duffing equations across many points of resonance. J. Diff. Eqns., 108, (1994), 152-169.

[24] J. L. Kazdan and F. W. Warner. Remarks on some quasilinear elliptic equations. Comm. Pure Appl. Math. 28, (1975), 567-597.

[25] M. G. Krein. On certain problems on the maximum and minimum of characteristic values and on the Lyapunov zones of stability. Amer. Math. Soc. Translations, series 2, vol. 1, Providence, R.I., 1955.

[26] S. Lang. Analysis.II. Addison-Wesley, Reading, Mass., 1969.

[27] A.C. Lazer. Application of a lemma on bilinear forms to a problem in nonlinear oscillations. Proc. Amer. Math. Soc., 33, (1972), 89-94.

[28] E.B. Lee and L. Markus. Foundations of Optimal Control Theory. Wiley, New York, 1967.

[29] Y. Li and H. Wang. Neumann boundary value problems for second order ordinary differential equations across resonance. SIAM J. Control Optim., 33, (1995), 13121325.

[30] M. A. Lyapunov. Problème général de la stabilité du mouvement. Ann. Fac. Sci. Univ. Tolouse Sci. Math. Sci. Phys., 2, (1907), 203-474.

[31] W. Magnus and S. Winkler. Hill's Equation. Dover, 1979.

[32] J. Mawhin and J. R. Ward, Nonuniform nonresonance conditions at the two first eigenvalues for periodic solutions of forced Liénard and Duffing equations. Rocky Mountain J. Math., 12, (1982), 643-654.

[33] J. Mawhin and J. R. Ward, Periodic solutions of some forced Liénard differential equations at resonance. Arch. Math (Basel), 41, (1983), 337-351.

[34] J. Mawhin, J.R. Ward and M. Willem. Variational methods and semilinear elliptic equations. Arch. Rational Mech. Anal., 95, (1986), 269-277.

[35] D. Smets, M. Willem and J. Su. Non-radial ground states for the Hénon equation. Comm. Contem. Math. 4, (2002), 1-14.

[36] G. Talenti. Best constant in Sobolev inequality. Ann. Mat. Pura Appl., 110 (1976), 353-372.

[37] H. Wang and Y. Li. Periodic solutions for Duffing equations. Non. Anal., 24, (1995), 961-979.

[38] M. Zhang and W. Li. A Lyapunov-type stability criterion using $L^{\alpha}$ norms. Proc. Amer. Math. Soc., 130, (2002), 3325-3333.

[39] M. Zhang. Certain classes of potentials for $p$-Laplacian to be non-degenerate. Math. Nachr., 278, (2005), 1823-1836.

Departamento de Análisis Matemático, Universidad de Granada, 18071 Granada, SPAIN.

E-mail address: acanada@ugr.es, svillega@ugr.es 